\title{
UNA MIRADA A LOS PLANES DE DESARROLLO DE CALDAS DESDE LOS OBJETIVOS DEL MILENIO. Una lectura subregional.
}

\section{A LOOK AT DEVELOPMENT PLANS IN CALDAS BASED ON THE MILLENNIUM DEVELOPMENT GOALS. A regional perspective}

José Fernando Olarte Osorio ${ }^{1}$

\section{Resumen}

No pretende este artículo evaluar el proceso de formulación de los planes de desarrollo, ni mucho menos su validez o su pertinencia. Tampoco pretende analizar datos, cifras o indicadores; la única pretensión es presentar un repaso a vuelo de pájaro de los contenidos de los Planes de Desarrollo de los entes territoriales del departamento de Caldas y la consideración que en estos se hace de los Objetivos del Milenio.

Si bien en todos pueden encontrarse programas, proyectos, metas o hechos que relacionan o suponen la apuesta que hay por avanzar en el logro de los objetivos, solo en algunos casos esto se hace de manera explícita. Hecho que se justifica en una sensación de rigidez en la estructura de los planes y en la poca sociabilización que de los Objetivos se ha hecho hacia instancias diferentes a las altas instituciones y a la academia.

Se espera que en el próximo proceso de planificación los Objetivos tengan una mayor consideración, pero es necesario acercar más a las comunidades a los propósitos que llevaron a su formulación y a las metas que persiguen, actividad que requiere el compromiso de todos quienes de alguna manera tenemos la responsabilidad autodelegada de trabajar por el logro de ellos.

\section{Abstract}

This paper does not pretend to assess the process, validity or relevance of designing development plans nor analyze their data or figures. The aim is to present a short overview of the development plan and their content of the territorial institutions in the state of Caldas and the discussion of their Millennium Development Goals - MDG.

In all development plans, we can find programs, projects, goals, or facts related to or involved with the proposal for making progress in the achievement of development goals. However, only some plans made explicit these aspects. This fact is evident in the rigidity of the structure in the development plan and the lack of public presentation of

1 Ingeniero de Sistemas; Especialista en Gestión Ambiental Local; Diplomado en Gestión Ambiental ISO 14000; Coordinador de Proyectos Programa Paz y Competitividad; Miembro del Grupo de Investigación en Desarrollo Regional de la UAM. Estudiante de la Maestría en Desarrollo Regional y Planificación del Territorio. 2008 - 2010.

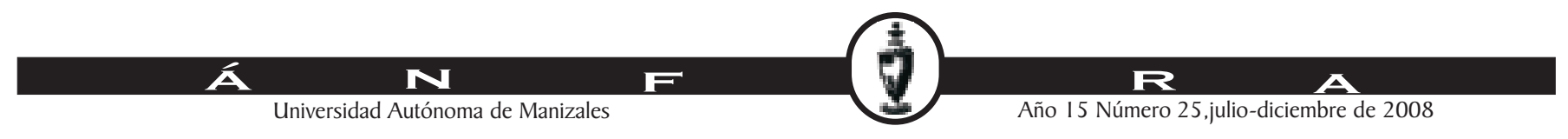


the development goals to/in institutions different from high government institutions and the academic world.

Even though it is expected that future planning processes will make an emphasis on the Millennium Goals, it is necessary to bring communities closer to their original purposes and the MDG pursued. This activity requires the commitment of all those involved and the responsibility to work in order to achieve them ${ }^{2}$.

\section{Consideraciones Iniciales}

El año 2008 es el inicio de un nuevo proceso político administrativo del país, es el año en que se normalizaron los periodos de alcaldes y gobernadores y sus respectivos concejos y asambleas. Particularmente, para el Departamento de Caldas, inician periodo simultáneamente el Gobernador y Alcalde de la capital, hecho que no sucedía con los últimos cinco mandatarios de la ciudad, los cuales habían sido elegidos en jornadas fuera de calendario, como también sucedió en el último periodo con Samaná, Filadelfia, Aguadas, Marmato y Viterbo, donde los alcaldes tuvieron un menor tiempo de gobierno.

Estos elementos tienen una incidencia enorme en el ejercicio de la planeación del Desarrollo de la región, ya que este año se posibilitaba la sincronización entre todos los gobernantes del departamento en sus esfuerzos para tener una agenda común de desarrollo, presupuestar proyectos de carácter subregional, ajustar las escalas de planeación con las visiones nacionales y departamentales; siendo el tema de la infraestructura vial, con toda razón y sin elemento que permita discusión alguna, una preocupación y propuesta de desarrollo común en todos los programas de gobierno que habían sido presentados por los candidatos a los cargos de elección popular que estaban en contienda.

Sumado a lo anterior, pero con la dificultad que implica la situación, el Departamento presenta una gran divergencia territorial y social entre sus subregiones y a su vez entre sus municipios; siendo el Oriente y la región del Magdalena la que concentra los mas preocupantes índices de desarrollo; sin que esto signifique que el resto de las subregiones se encuentran en posiciones privilegiadas.

A manera de referente se presentan algunos datos estadísticos, tomados de la Carta Estadística del Departamento, insumo para la formulación del plan de desarrollo departamental.
2 Resúmenes traducidos por el gestor de traducciones del Instituto de Idiomas de la UAM, Carlos A. Muñoz Torres, Máster en Teoría de la Traducción, Universitat Autònoma de B a r c elona.Resúmenes revisados por Kevin Guzzo, nativo del inglés, estudiante de la Maestría en Traducción de la Universidad Autónoma de Manizales.

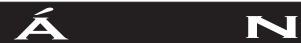

Universidad Autónoma de Manizales 
Proyección de población del departamento por subregiones

\begin{tabular}{|c|c|c|c|c|c|c|c|c|c|}
\hline \multirow{2}{*}{ Dentis } & \multicolumn{3}{|c|}{$-1=$} & \multicolumn{3}{|c|}{$=$} & \multicolumn{3}{|c|}{20} \\
\hline & $\frac{\ln }{n i n}$ & $\sin 2$ & nim & 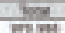 & intirs & ans & $\frac{5}{m}$ & sfetes & 글 \\
\hline 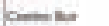 & Etwr & mos & tom & $\cos m$ & $m=$ & $n n a$ & mean & 6 & nam \\
\hline penem & Wo16? & 2030 & $x=$ & $\bar{w} \omega$ & wes: & $\mathrm{min}$ & wout & $=2$ & and \\
\hline bentin & $\sin$ & $=70$ & 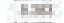 & $\mathrm{n}=$ & ines & 243 & was & then & $\operatorname{lan}$ \\
\hline$=$ & min & num & $\ln 00$ & pain & $10 \mathrm{y}$ & uan & asti & was & then \\
\hline Cants. & 750 & ars & 1zan & ir & $=$ & t1500 & trat & $3 \pi$ & 24 \\
\hline nowes & $n=$ & wing & ven & enth & $m a n$ & near & atson & $m e$ & \\
\hline min & $\pi$ m & nin & Num & nam & Nen & $m n$ & $n e$ & med & $x=$ \\
\hline Reand & $a x$ & natent & нะi & $a m$ & $n=$ & wita & Dasu & EDas & ant \\
\hline Nomet & ans & $\sin$ & sae & $6 x$ & $r=$ & ams & $\operatorname{tras}$ & $\operatorname{trn}$ & \\
\hline$m$ & this & $i e$ & In & iest & int & MSE & кек & 100 & SHE \\
\hline 10 & min & $n=$ & ine & $6=0$ & $N=$ & tina & neap & $n=$ & rese \\
\hline 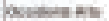 & ating & Hein & Hatl & texin & mont & niw & wits & $=0$ & mim \\
\hline romb & सात & this & $\sin$ & $\tan x$ & 50 & Atat & Itamt & 1.3e & sin \\
\hline unsent & she & $i=$ & ane & 100 & LT & Ant & in & $i=x$ & s. Me \\
\hline motes: & $=1+\infty$ & $11+5$ & 10 & 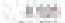 & 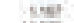 & tise & 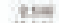 & 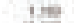 & $2 x^{2}$ \\
\hline Ponese & 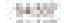 & wen & $=04$ & $\times 4$ & iss. & $=2$ & 806 & $\neq \infty$ & ext4 \\
\hline nom & ine? & $n \mathrm{~m}$ & ens & 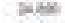 & $n=0$ & Hent & 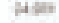 & $n=$ & 12046 \\
\hline povitere $8=$ & $\tan$ & At that & Hese & $m n$ & $4 t=$ & inase & main & et & winf \\
\hline weos: & $n=$ & nosu & $10 \times$ & $=\infty$ & Ds & $\mu x$ & $x y$ & In & wos \\
\hline penoms & Hin & $1 / 2$ & ese & N30 & $5 m$ & $\cos$ & +5 & tas & 1500 \\
\hline pent: & $\operatorname{tin}$ & Afi & 10 & 640 & tes & 47 & stit & tax & in \\
\hline $\ln =$ & tas? & iat & 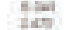 & 7 in & is & san & $r=$ & tein & ingt \\
\hline Lasoter & tont & anter & และ & tars & 14: & 4 & sets & 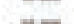 & \\
\hline Anstar & the & $1=4$ & $=0$ & $w i$ & $\sin$ & wis & sin & $=$ & 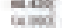 \\
\hline mosents & whe & fin & kit? & Fon & $s=$ & $6 \mathrm{mit}$ & int & in & . \\
\hline Henes & ste & itie & $a=i$ & ith & $k=$ & in & we & $1=$ & and \\
\hline puse & sisis & $\therefore$ & 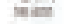 & $=2$ & 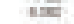 & niti & $a m$ & $i=$ & mate \\
\hline thenters & & & & & & & & & \\
\hline pens & Hene & Hint & inst & Tetin & 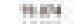 & nat & msen & $m$ & war \\
\hline Goomin & have & none & Wu & sen & nest & $1+0$ & and & miv & $y=$ \\
\hline poses & 네료 & in & sfir & in & $t=$ & iset & sive & 새료 & 3 幽 \\
\hline $\operatorname{lones}$ & בati & ing & zan & 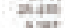 & $4=$ & in & Dize & 10 & axt \\
\hline thens & stim & In. & 130 & $s=t$ & $15=$ & Ang & $\ln$ & 12 & \\
\hline
\end{tabular}

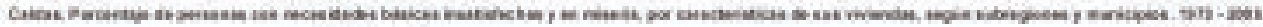

\begin{tabular}{|c|c|c|c|c|c|c|c|c|c|c|c|c|c|c|c|c|c|c|c|c|c|c|c|c|}
\hline \multirow[t]{2}{*}{ Denesery } & \multicolumn{4}{|c|}{ 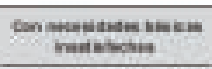 } & \multicolumn{4}{|c|}{ 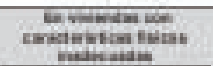 } & \multicolumn{4}{|c|}{ 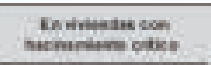 } & \multicolumn{4}{|c|}{ 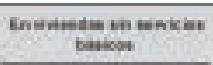 } & \multicolumn{4}{|c|}{ 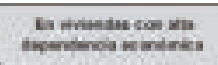 } & \multicolumn{4}{|c|}{ 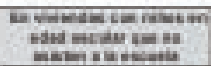 } \\
\hline & 5 & Was & $\mathrm{sen}$ & $a=$ & $m$ & 101 & $\tan$ & $\mathrm{an}$ & 90 & the & $\tan$ & Exis & $\operatorname{mon}$ & sesi & than & 2006 & $\mathrm{~m}$ & tas & end & an & $m n$ & tons & 100 & 1000 \\
\hline net: & nis & $n:$ & 33 & 바 & wa & 20 & 24 & 15 & $8 s$ & 03 & ass & siz & $\mathbf{3 2}$ & 2. & 35 & 13 & $2 x x$ & 756 & 120 & na & as & 43 & 7.8 & $\mathrm{~m}$ \\
\hline Camsas & mo & A. & 92 & 2.2 & Cs: & a) & n,o & ay & as & $\pm x$ & 20 & we & 42 & 20 & 12 & 18 & 2as & at & at & $a$ & an & $x_{4}$ & 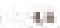 & \\
\hline & & & & & & as & as & & & & & & & L & & & & & & & & & & \\
\hline Cheots & 80 & 3.2 & 87 & 169 & $2+2$ & a. & +8 & 25 & जा & 65 & 140 & is & 4.4 & 32 & 1 & & 320 & $12 A$ & is & 62 & inz & is & B. & $2 x$ \\
\hline$\Leftrightarrow$ & an & an: & ato & ans. & $w$ & L & ta & 20 & as & 7.1 & nis & 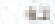 & 172 & a. & 27 & & 123 & 22. & tas & Ga & e.: & b) & tas & in \\
\hline resen & ast & 2 & Ws & $a b$ & 570 & 6 & 6 & 26 & $x_{2} 2$ & 2 & ist & 20 & 65 & 13 & 4 & a & 303 & 20 & 15 & os & 33 & wis & $\pi, 8$ & 36 \\
\hline $\operatorname{lin}$ & ses & a) & is & nia & $\$ 2$ & as & 2 & as & 3.4 & ws & is & 29 & 103 & 35 & id & $z$ & 323 & 31 & is & 62 & 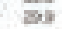 & as & 13 & \\
\hline Nirs & ara & D. & $=44$ & 218 & ra & 24 & ax & $a t$ & 2. & $\approx 0$ & 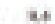 & 4 & 174 & $=$ & sas & as & 328 & 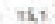 & $\mathrm{na}$ & 44 & at4 & wht & nts & \\
\hline Noine & nat & as & is & 2 & 25 & 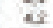 & 20 & & as, & 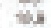 & 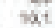 & i & a? & wis & & & 60 & 22 & 52 & is & st & 4.1 & 125 & \\
\hline & & & & & & 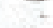 & as & & & & & & & E. & & & & & & & & & & \\
\hline ners & $\vec{x} ;$ & Es & 83 & 209 & $k T$ & 25 & is & 02 & 8.4 & क) & at & Ix & 124 & aiv & is & & $32 \pi$ & 172 & 8.3 & ถอ & $\infty 2$ & wa & Ha & \\
\hline neatres & ind & ais & aेs & 164 & 2 & is & 3 & ai & as & M & is & 5 & 124 & C. & 4 & $\vec{w}$ & nit & 107 & Re & Ma & ai & su & 11 & \\
\hline & & & & & & & as & & & & & & & 82 & & & & & & & & & 6 & \\
\hline Fall & $=$ & wa & is & 256 & $\$ 1$ & 10 & $\underline{y}$ & ca & ses & कs & Nis & 4 & ins & xis & 4 & is & ier & 10 & an & an & its & $\pi$ & 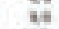 & i. \\
\hline Lav & rat & a. & ths & as & $\omega$ & 25 & ato & & & 67 & $n+1$ & 2 & & bia & $z$ & & & & & . & & a, 1 & $\pi \mathrm{s}$ & \\
\hline var & ris & +13 & hs & w & tas & $n$ & $\mathrm{H} 2$ & & & 8 & wa & 120 & & (i) & & $n \pi$ & & & & - & & & it & \\
\hline Kine & मA & se. & बis & & & in & $\$$ & & & : & 50 & 20 & & w & & & & & & sit & & & ii & \\
\hline son & 79 & Mi & na & aw & noa & $n z$ & $\vec{M}$ & 28 & $\approx$ & w. & ar & is & $x 4$ & s. & as & 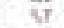 & ins & 228 & 7,7 & $\theta y$ & ms & wix & is & \\
\hline denes & & & & & & 4 & 25 & & & tot & $\pi 4$ & 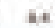 & & $=$ & 24 & & & & & & & & 124 & t. \\
\hline & & & & & & & & & & & & & & & & & & & & 6.) & & & & \\
\hline se & ng & w. & 82 & 3 & 40 & s. & a & & & 49 & uss & is & 14 & क) & 33 & & & 125 & 122 & & $y$ & 203 & 47 & \\
\hline & no & a? & Ho & & & a & 22 & & & & & & $3 n$ & e) & 21 & & ita & 145 & ago & & 5 & ir & 124 & \\
\hline & & & & & & & & & . & . & . & i & & & 21 & & & . & . & & . & mo & . & \\
\hline ก.า & e.1 & ast & no & 25 & 222 & su & 25 & so & ex & 4.4 & na & 420 & $x$ & $\mathbf{3 4}$ & 13 & ar & ns & 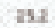 & 140 & as & nos & we. & 94 & 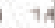 \\
\hline & & & & & & a & 11 & & & & & & & & & & & & & & & & 121 & 16 \\
\hline & 4 & 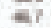 & i & 2 & & 20 & $\omega$ & & & & & & & s & & & & a. & Ba & as & & w & 124 & th \\
\hline & $\pi$ & & & & & a & & & & & & & & & & & & & & & & & & \\
\hline & $\mathrm{sin}$ & 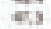 & & & & 25 & as & & & & & & & z & & & & & & & & & & \\
\hline Nontian & ati & no & wo & ase & as & & as & & is & wi. & $2 \pi x$ & 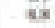 & $10 \%$ & s. & $x 4$ & $\omega$ & ins & 120 & 16. & $\pm x$ & as & 24 & 126 & 20 \\
\hline & & & & & & & & & & & & & & Das & & & & & nat & & & 115 & at6 & 26 \\
\hline & a & 4 & ns & & & 8 & & & & & & & & & & & & 3 & iss & & & $\omega_{2}$ & is & \\
\hline & & & & & & & & 12 & & & & & & & & & & & & & & & & \\
\hline & wa & 40 & 5 & ats & & $+x \mid$ & 73 & & & 84 & & 12 & & & & & & 00 & 8 & $=$ & ais & 26.3 & 149 & \\
\hline & an & wa & 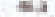 & 23 & 6 & wes & 10. & & wi. & w.7 & 134 & 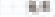 & A.t. & $>0$ & $=a$ & & 40.7 & $18 \pi$ & RA & $=$ & If & $=y$ & 120 & \\
\hline
\end{tabular}

be

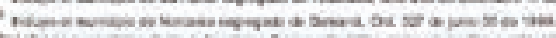

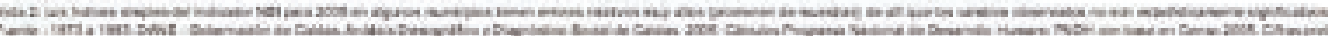

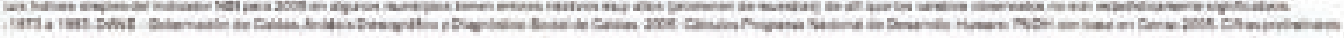

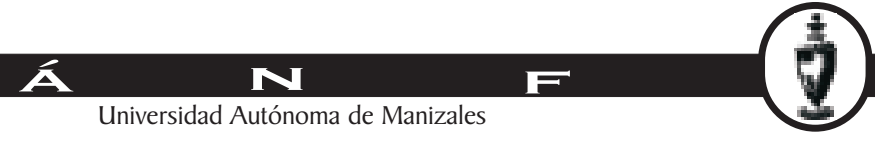


Caldas. Población identificada en el SISBEN por nivel, según municipios. A marzo de 2007

\begin{tabular}{|c|c|c|c|c|c|c|c|c|c|}
\hline \multirow{2}{*}{$\begin{array}{l}\text { Distritos y } \\
\text { Municipios }\end{array}$} & \multirow[t]{2}{*}{ Total } & \multicolumn{8}{|c|}{ Nivel SISBEN } \\
\hline & & 1 & $\%$ & 2 & $\%$ & 3 & $\%$ & 40 más & $\%$ \\
\hline Caldas & 702.848 & 219.471 & 31,2 & 349.353 & 49,7 & 129.083 & 18,4 & 4.941 & 0,7 \\
\hline Centro Sur & 322.172 & 93.116 & 28,9 & 151.869 & 47,1 & 74.244 & 23,0 & 2.943 & 0,9 \\
\hline Manizales & 203.499 & 54.333 & 26,7 & 90.257 & 44,4 & 56.964 & 28,0 & 1.945 & 1,0 \\
\hline Chinchiná & 46.081 & 18.704 & 40,6 & 22.409 & 48,6 & 4.567 & 9,9 & 401 & 0,9 \\
\hline Neira & 23.890 & 4.504 & 18,9 & 13.511 & 56,6 & 5.708 & 23,9 & 167 & 0,7 \\
\hline Palestina & 22.987 & 7.266 & 31,6 & 13.738 & 59,8 & 1.750 & 7,6 & 233 & 1,0 \\
\hline Villamaría & 25.715 & 8.309 & 32,3 & 11.954 & 46,5 & 5.255 & 20,4 & 197 & 0,8 \\
\hline Norte & 73.118 & 20.491 & 28,0 & 44.103 & 60,3 & 8.296 & 11,3 & 228 & 0,3 \\
\hline Aguadas & 23.742 & 6.207 & 26,1 & 15.386 & 64,8 & 2.112 & 8,9 & 37 & 0,2 \\
\hline Aranzazu & 13.945 & 5.154 & 37,0 & 7.300 & 52,3 & 1.447 & 10,4 & 44 & 0,3 \\
\hline Pácora & 15.560 & 2.811 & 18,1 & 10.722 & 68,9 & 1.939 & 12,5 & 88 & 0,6 \\
\hline Salamina & 19.871 & 6.319 & 31,8 & 10.695 & 53,8 & 2.798 & 14,1 & 59 & 0,3 \\
\hline Occidente Alto & 67.469 & 16.253 & 24,1 & 35.554 & 52,7 & 15.241 & 22,6 & 421 & 0,6 \\
\hline Filadelfia & 11.980 & 3.042 & 25,4 & 7.020 & 58,6 & 1.844 & 15,4 & 74 & 0,6 \\
\hline La Merced & 6.370 & 1.620 & 25,4 & 3.876 & 60,8 & 843 & 13,2 & 31 & 0,5 \\
\hline Marmato & 8.185 & 3.183 & 38,9 & 4.337 & 53,0 & 647 & 7,9 & 18 & 0,2 \\
\hline Riosucio & 15.643 & 2.831 & 18,1 & 5.829 & 37,3 & 6.825 & 43,6 & 158 & 1,0 \\
\hline Supía & 25.291 & 5.577 & 22,1 & 14.492 & 57,3 & 5.082 & 20,1 & 140 & 0,6 \\
\hline Occidente Bajo & 75.384 & 24.040 & 31,9 & 37.017 & 49,1 & 13.857 & 18,4 & 470 & 0,6 \\
\hline Anserma & 33.760 & 11.789 & 34,9 & 15.710 & 46,5 & 6.128 & 18,2 & 133 & 0,4 \\
\hline Belalcázar & 11.018 & 3.955 & 35,9 & 5.643 & 51,2 & 1.384 & 12,6 & 36 & 0,3 \\
\hline Risaralda & 11.293 & 2.502 & 22,2 & 6.315 & 55,9 & 2.327 & 20,6 & 149 & 1,3 \\
\hline San José & 5.131 & 1.265 & 24,7 & 3.412 & 66,5 & 427 & 8,3 & 27 & 0,5 \\
\hline Viterbo & 14.182 & 4.529 & 31,9 & 5.937 & 41,9 & 3.591 & 25,3 & 125 & 0,9 \\
\hline Alto oriente & 60.940 & 19.283 & 31,6 & 35.883 & 58,9 & 5.467 & 9,0 & 307 & 0,5 \\
\hline Manzanares & 20.224 & 6.765 & 33,5 & 11.939 & 59,0 & 1.479 & 7,3 & 41 & 0,2 \\
\hline Marquetalia & 14.606 & 3.360 & 23,0 & 9.112 & 62,4 & 2.101 & 14,4 & 33 & 0,2 \\
\hline Marulanda & 3.123 & 1.892 & 60,6 & 1.094 & 35,0 & 137 & 4,4 & - & - \\
\hline Pensilvania & 22.987 & 7.266 & 31,6 & 13.738 & 59,8 & 1.750 & 7,6 & 233 & 1,0 \\
\hline $\begin{array}{l}\text { Magdalena } \\
\text { Caldense }\end{array}$ & 109.754 & 47.779 & 43,5 & 49.902 & 45,5 & 11.714 & 10,7 & 336 & 0,3 \\
\hline La Dorada & 70.664 & 28.290 & 40,0 & 32.942 & 46,6 & 9.116 & 12,9 & 316 & 0,4 \\
\hline Norcasia & 7.057 & 4.047 & 57,3 & 2.616 & 37,1 & 387 & 5,5 & 7 & 0,1 \\
\hline Samaná & 22.551 & 10.276 & 45,6 & 10.947 & 48,5 & 1.315 & 5,8 & 13 & 0,1 \\
\hline Victoria & 9.482 & 5.166 & 54,5 & 3.397 & 35,8 & 896 & 9,4 & - & - \\
\hline
\end{tabular}

Fuente: Secretaría de Planeación Departamental con base en base de datos SISBEN validada por DNP

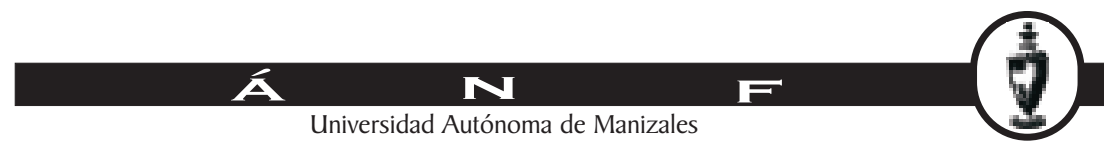




\begin{tabular}{|c|c|c|c|c|c|c|c|c|c|c|c|c|}
\hline \multirow{2}{*}{ bubley } & \multicolumn{3}{|c|}{ 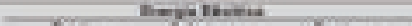 } & \multicolumn{3}{|c|}{ Noterated } & \multicolumn{3}{|c|}{$x=-\sin$} & \multicolumn{3}{|c|}{ 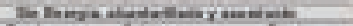 } \\
\hline & $x=$ & Gavente & Sentes & Mave & Cestan & Ant & tow & Getativa & hents & & Contitis. & hat \\
\hline & ब⿳亠口冋口2 & wes & in & WU & कार & an & 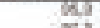 & min & nis & 25 & 03 & 매 \\
\hline Mewases & as & ns & mis & at & na, & ase & $\operatorname{sex}$ & atz & $=4$ & as & a & ae \\
\hline onime. & as & n: & $=3$ & min & m & ace & mi & at & nis & at & a & 녀 \\
\hline$\rightarrow$ & $2:$ & $m$ & ki & 723 & me & we & 20 & at & 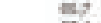 & 13 & $a 2$ & 2a) \\
\hline nemil & 0.2 & an & wat & 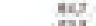 & w1 & mas & 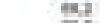 & a! & et. & M & a) & 24: \\
\hline Mesis & es & m. & Ma & 53 & $m a$ & ris. & as & ni & Na & a) & a). & 2u \\
\hline $\mathrm{men}$ & 27 & nas & wa & $6 y$ & wo & su & not & m! & ses & $\mathbf{3}$ & a) & 24 \\
\hline tanise & wa & $m$ & N13 & $*$ & ats & us & hes & whit & $=2$ & a & as & 29 \\
\hline Nowald & 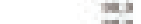 & $m$ & $=1$ & 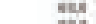 & $m$ & $22 \pi$ & nit & at & 42 & In & at & 22 \\
\hline Nara & 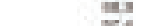 & nis & $=2$ & $\mathrm{~m}$ & $m$ & ant & nin & teta & ad & 28 & A1 & 20 \\
\hline Aannos & $=14$ & $m x$ & $=1$ & $m$ & $m$ & at & ma & at: & as & 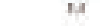 & 23 & 13. \\
\hline audtents as & ses & $\mathbf{n n}$ & we & $n a$ & mo & ma & ax & wa & na & 14 & 9 & 24 \\
\hline nlasto & 9.9 & ns & si & 74 & 20 & 340 & $\mathrm{~ns}$ & 4 & w2 & 13 & 26 & 24 \\
\hline atencet & $\infty 3$ & ns & nit & $4 \sqrt{14}$ & ni, & tas & ces & Nit & $=5$ & is & 21 & 헤 \\
\hline Bone: & ins & a.t. & Wh & 42 & tab & 24 & 24 & \#i & is & हs & 24 & ज) \\
\hline howes & in: & as & ing & $\mathrm{N} 24$ & ites & as & int & ints & $M A$ & L & at & 21 \\
\hline seis & बin & $\bar{n}$ & in & 72,1 & 36 & net & 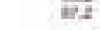 & nis & Nas & น & a) & 29 \\
\hline aicebro ans & $m a$ & na & ota & $\operatorname{tas}$ & Ites & ine & nea & $\Rightarrow$ & as & 13 & at & u \\
\hline Aven & 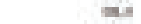 & $=$ & it: & tax & $m$ & and & $\pi, r$ & ats & as & $\mathrm{ma}$ & as & it \\
\hline Anowater & $\infty 4$ & $m$ & $=1$ & $\operatorname{lag}$ & $\sec$ & $42 \pi$ & $\pi, n$ & $\mathrm{ma}$ & $=$ & 13 & as & In \\
\hline carwas & is & $\Rightarrow$ & w, & ast & $m$ & +28 & ut & at & $\geqslant ;$ & 1.4 & ai & u \\
\hline $\operatorname{ande}$ & an & $n=$ & $=9$ & $\operatorname{san}$ & mo & and & an & nty & sen. 1 & is & as & 2at \\
\hline$=1$ & an & $m$ & $\mathrm{ma}$ & ma & ats & ase & $\min$ & $m$ & का & 12 & a & a. \\
\hline ats ond & acs & $=1$ & $=4$ & 271 & $a k, 1$ & ant & me & $=1$ & $=9$ & 17 & as & an \\
\hline therers & Qs & es & 20 & sid & 125 & in & (1) & Not & 12 & is & as & 24 \\
\hline Hrevera & as & nes & His & $\omega$ & we & \&) & 63 & at: & 3.4 & 2 & an & is \\
\hline Menisits & ws & $a_{2}$ & $m$ & 34 & ins & 06 & ins & mit & sent & E. & at & a \\
\hline 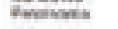 & ins & in & $=2$ & 54 & nit & B. & $\$ 26$ & ar & nis & 12 & 2 & v \\
\hline matames: & & & & & & & & & & & & \\
\hline cinast. & 97 & p: & $=5$ & $2 \mathrm{~N}$ & 204 & as & 93 & 6: & sti. & s & 12 & 10.4 \\
\hline Goonte & as: & os & 203. & sN & as & 321 & $\alpha_{2}$ & कs & 692 & 63 & af & 4 \\
\hline nisas & ans & $=a$ & $\sin$ & est & $m=$ & $\mu$ & 125 & 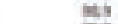 & $n$ & ax & ab & in \\
\hline Smon: & 48 & v.s. & es & $4 \mathrm{M}$ & 94 & 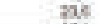 & sts & al & 57 & 81 & IA & 119 \\
\hline ens: & QS: & ns & in & 58 & 134 & ind & in & $\mathrm{si}$ & s) & $\mathrm{sa}$ & as & ind \\
\hline
\end{tabular}

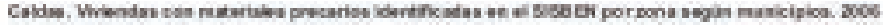

\begin{tabular}{|c|c|c|c|c|c|c|c|c|c|c|c|}
\hline \multirow[b]{2}{*}{ Deniden } & \multirow[b]{2}{*}{ Now onineds } & \multicolumn{4}{|c|}{ 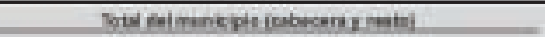 } & \multicolumn{3}{|c|}{ Catechia mentupai } & \multicolumn{3}{|c|}{ 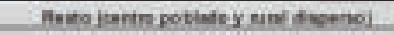 } \\
\hline & & $\begin{array}{l}\text { Vwandas } \\
\text { siseck }\end{array}$ & 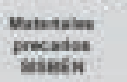 & $\begin{array}{l}\text { soon roikisin } \\
\text { insty }\end{array}$ & 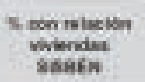 & $\begin{array}{l}\text { Yivesale } \\
\text { suseck }\end{array}$ & 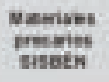 & $\begin{array}{c}\text { tean reacien } \\
\text { minem } \\
\text { sisobi }\end{array}$ & Mimelan & 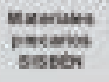 & 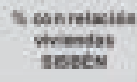 \\
\hline Calen & 20083 & $\sin \times 10$ & $7524 \mathrm{f}$ & 39 & 92 & 170350 & 2.01 & 46 & 5530 & 5.1 .45 & 10,1 \\
\hline Getn Sx & tasin & 71.545 & 265: & 20 & St & stae & 2.45 & $4 A$ & Mas & 1283 & ta \\
\hline Mavalet & tacent & 2. 10 & $2 \sin$ & 25 & 5,7 & 32312 & 2005 & 5.1 & 1990 & $\$ 52$ & 2,0 \\
\hline Cawhes & ts $3 x$ & ani & $25 s$ & 23 & 26 & $7 \times 206$ & ma & 21 & 230 & w3 & 4,3 \\
\hline Netr & asis & 0.06 & $2 n$ & 12 & 30 & 272 & 34 & 12 & 2069 & 175 & 5,7 \\
\hline Notina & 2000 & 4.20s & $w$ & 24 & 2.5 & 2415 & $n$ & 29 & Mas & $3 ?$ & 2,2 \\
\hline vilarutis & 12000 & a.s & 45 & 19 & Ts & twit & 100 & t.t & 295 & 294 & 56 \\
\hline heres & $\operatorname{tash}$ & 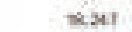 & 45 & 25 & $2 y$ & $2 \sin$ & w3 & 12 & $25 s$ & 33 & 3,3 \\
\hline Aousten & 648 & $6+\infty$ & N & 8 & 13 & 2ats & s & $a g$ & 1360 & ss & 1,9 \\
\hline Amexts & 205 & 3.400 & "1: & u. & is & $2 \mathrm{win}$ & $n$ & 29 & ISn & $A ?$ & 2.0 \\
\hline Pavas & 4.186 & $+x=0$ & $=$ & 5 & 13 & 200 & 3 & as & 360 & is & 30 \\
\hline 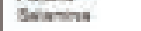 & 5500 & $5 \times 00$ & $2 \bar{x}$ & is & 46 & 2015 & a & 27 & 200 & तi & 22 \\
\hline becidenes wie & $243 x$ & Ir, th & $2 \mathrm{x}$ & as & +2 & $a \approx 6$ & ss & $3 z$. & $7.2 x$ & 149 & 1.9 \\
\hline Fioseta & 335 & 3.00 & $N$ & 2.2. & 23 & 200 & s & $a 9$ & 2300 & 44 & 21 \\
\hline La Morod & 1.000 & (.)1 & wa & $\$, 1$ & 12 & 124 & $\pi$ & $\alpha_{2}$ & var & is & 1,0 \\
\hline Martats & $\tan$ & t.2er & w & as & $a$. & $2 k$ & 3 & t. & 200 & a & M \\
\hline Natersa & 14.431 & 4.417 & si & $a_{4}$ & 13 & 439 & 47 & 12 & 360 & $\boldsymbol{n}$ & 2.7 \\
\hline sen & an & ara & 4 & $a 7$ & $a$ & करा & 4 & as & 2.80 & 42 & 1,4 \\
\hline bosudene teos & $10 b a 4$ & trase & 30 & to & 2.) & $2 \mathrm{me}$ & $2 n$ & 13 & 7,190 & 347 & a.t \\
\hline Anesma & 200 & a.210 & 16 & 19 & is & $4 \sin$ & 3 & ata & 2030 & 13? & 2.3 \\
\hline Devedas & 200 & 2000 & $\mathrm{n}$ & 12 & is & 2246 & 43 & 35 & 243 & is & 3.9 \\
\hline Rervita & 2140 & 2.570 & $3 \pi$ & 32 & s.t. & 1315 & 24 & 24 & 155 & 43 & 4 \\
\hline Snviat & t.ros & 1.213 & $x$ & is & 2,1 & $33 r$ & 3 & $2 x$ & 900 & $v$ & 1.3 \\
\hline keos & 3200 & save & $\bar{z}$ & $a$ & ad & $2 \pi 00$ & s & as & 40 & 1 & 4 \\
\hline ano oints & rasp & Theses & sast & 38,7 & 4he & $1 \times 0$ & 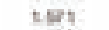 & $\boldsymbol{x}, t$ & 146 & 3473 & w. \\
\hline Mareanxist & arent & tine & 250 & 224 & nat & 201 & 302 & 120 & 2535 & 1211 & as \\
\hline waryeres. & $\operatorname{son}$ & $8 \times 58$ & Nas & nas & $2 t 5$ & 155 & 193 & 20 & 239 & 344 & 30 \\
\hline Mariands & $a=$ & Mo & 35 & 552 & Dá & 252 & 53 & $\$ 62$ & 970 & 43 & n.s. \\
\hline Novileava & $\sin$ & 607 & $4+\pi$ & 22.3 & srin & 174 & $\tan$ & $5 \pi, T$. & t) & 2.945 & ne \\
\hline \multicolumn{12}{|l|}{ enotyles: } \\
\hline Ematus & mase & thiass & 250 & He & $12 y$ & 59.81 & ๓? & it. & $7.14 \pi$ & 2343 & 3.s. \\
\hline wosesh & $100 x$ & $x \times 0$ & in & $\mathrm{xt}$ & 36 & ts 100 & 25 & $t 7$ & ta2 & ass & Dอ \\
\hline Norans & 1.799 & 1.000 & 39 & 223 & 31.1 & 197 & ta & tos & $5=$ & 230 & sis \\
\hline gemans & $\operatorname{aen} 1$ & 5.500 & $20 \%$ & ing & Iis. & 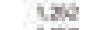 & azs & tis & $42 n$ & 1947 & $42 z$ \\
\hline yousa & 2000 & 2071 & $3 \times$ & 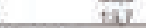 & is & 182 & 24 & 21 & 220 & 332 & 25 \\
\hline
\end{tabular}

Se suma a lo anterior, la difícil situación fiscal y financiera de los municipios, los cuales, con excepción de la capital, están clasificados en sexta categoría

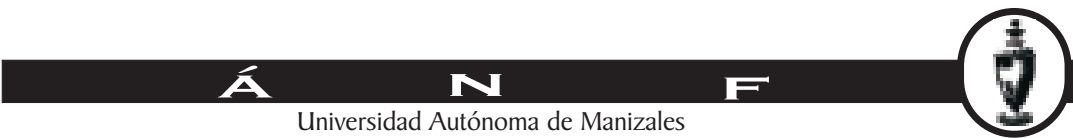




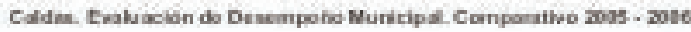

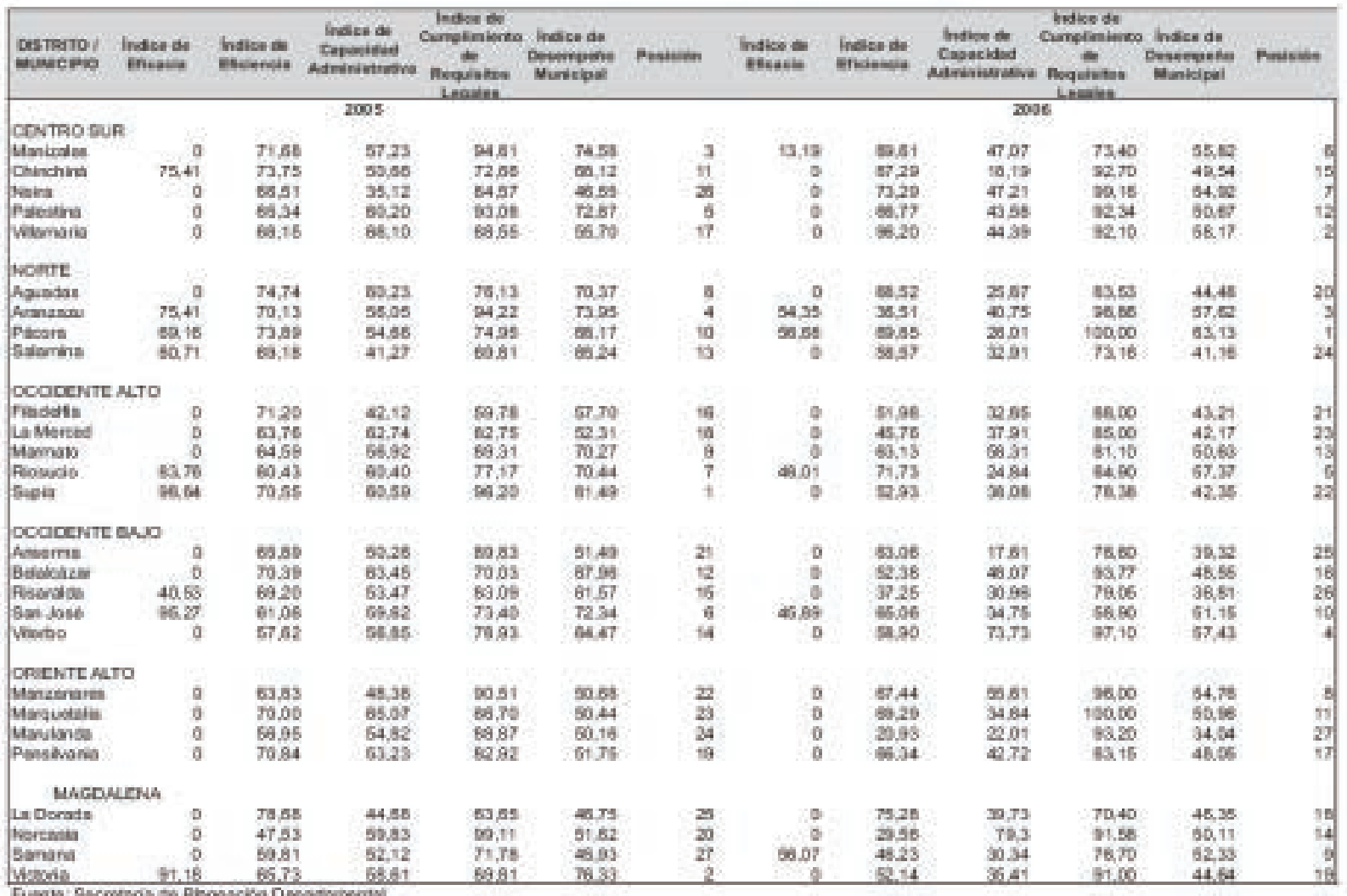

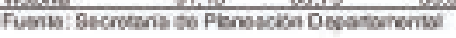

Con un escenario, como el ligeramente esbozado en los datos anteriormente suministrados, tenían los 27 alcaldes y el gobernador, la responsabilidad de formular en cuatro meses el Plan de Desarrollo Municipal y Departamental.

Para lograr el anterior objetivo, la Gobernación programó un encuentro por cada subregión para socializar sus propósitos, escuchar y poner en discusión los intereses de las comunidades. Por su parte, cada Alcaldía dispuso de los recursos, equipos y personal, de la manera que consideró conveniente y pertinente para realizar el ejercicio; quizás hubiese sido muy conveniente y oportuno un mayor acompañamiento por parte de la Secretaría de Planeación, pero esto no es materia de análisis y discusión en este artículo.

Contaron los municipios del Oriente con la oportunidad de recibir un acompañamiento de la Agencia de Cooperación Alemana GTZ para la formulación de sus planes de Desarrollo con un enfoque de Paz; esto les permitió tener un equipo de asesores permanentes en el proceso; situación que los municipios del resto de las subregiones debieron asumir, bien fuera a través de la contratación de asesores externos ó delegando estas funciones en equipos conformados por personal de la administración municipal.

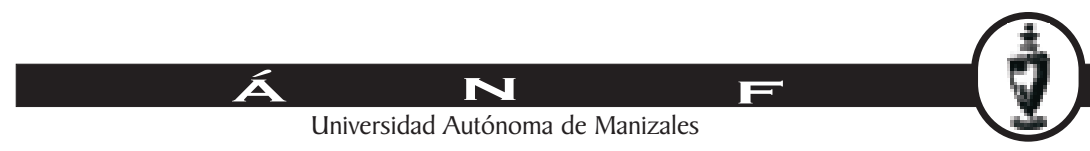


Una dificultad común, expresada por muchos de quienes de una u otra manera participamos en la formulación de planes de desarrollo municipales, fue la dificultad para acceder a información de línea base; no se contaba en la mayoría de los casos con sistemas de información actualizados o de fácil y masivo acceso y lectura.

Finalmente, todos los Planes de Desarrollo Municipal fueron aprobados a través de un proyecto de acuerdo en cada Concejo Municipal, con excepción del de Riosucio que debió ser decretado por el Alcalde. También pasó, por aprobación en la Asamblea, el plan de Desarrollo Departamental.

I gualmente se presentaron multiples requerimientos de incorporación de elementos, procesos, programas o actividades por parte de entidades del ámbito nacional, que terminaban afectando las asignaciones presupuestales

\section{ODM en el Departamento}

Caldas fue uno de los Departamentos seleccionados por el PNUD para adelantar un levantamiento de línea base de los indicadores que permitiesen evaluar y hacer seguimiento a los objetivos del Milenio. Este ejercicio fue desarrollado por el Centro de Estudios Ambientales y de Desarrollo de la Universidad Autónoma de Manizales, basándose metodológicamente en el levantamiento y análisis de informacion secundaria. Los resultados de este ejercicio fueron presentados en el tiempo en que se estaban formulando los planes de desarrollo; sin embargo, en algunos casos, se presentaron reparos entre la información presentada y la que los municipios evidenciaban o percibían (lo que reafirma la dificultad expresada por la falta de información confiable para el establecimiento de línea base).

A manera de referencia y con el ánimo de presentar los indicadores propuestos, se presenta el cuadro de información municipalizado construido por el CEA en su estudios, del cual no se hace un análisis ya que no es el objetivo de este artículo.

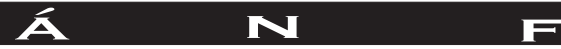

Universidad Autónoma de Manizales 


\begin{tabular}{|c|c|c|c|}
\hline \multirow[b]{2}{*}{$\begin{array}{l}\frac{\tilde{O}}{0} \\
\frac{\mathrm{U}}{2} \\
\frac{5}{\Sigma}\end{array}$} & \multicolumn{3}{|c|}{ PERFIL DEPARTAMENTAL } \\
\hline & 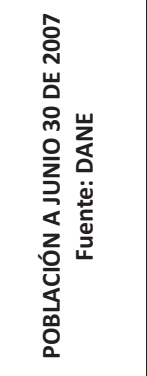 & 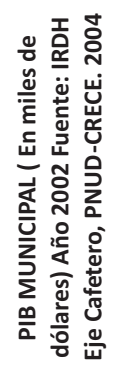 & 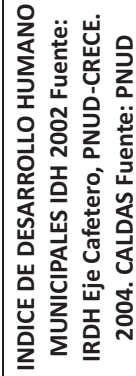 \\
\hline Manizales & $385.343,00$ & 852.004 & 0,778 \\
\hline Aguadas & $23.745,00$ & 18.017 & 0,623 \\
\hline Anserma & $34.818,00$ & 24.672 & 0,668 \\
\hline Aranzazu & $12.624,00$ & 6.391 & 0,640 \\
\hline Belalcázar & $11.565,00$ & 8.493 & 0,631 \\
\hline Chinchiná & $54.019,00$ & 51.613 & 0,654 \\
\hline Filadelfia & $12.377,00$ & 8.136 & 0,648 \\
\hline La Dorada & $74.395,00$ & 40.008 & 0,645 \\
\hline La Meced & $6.389,00$ & 4.451 & 0,628 \\
\hline Manzanares & $24.775,00$ & 10.235 & 0,620 \\
\hline Marmato & $8.596,00$ & 3.346 & 0,654 \\
\hline Marquetalia & $14.860,00$ & 8.110 & 0,634 \\
\hline Marulanda & $3.418,00$ & 1.215 & 0,568 \\
\hline Neira & $28.079,00$ & 11.953 & 0,628 \\
\hline Norcasia & $6.524,00$ & * & $*$ \\
\hline Pácora & $14.623,00$ & 10.946 & 0,671 \\
\hline Palestina & $17.947,00$ & 18.570 & 0,641 \\
\hline Pensilvania & $26.375,00$ & 11.439 & 0,637 \\
\hline Riosucio & $55.019,00$ & 17.391 & 0,631 \\
\hline Risaralda & $10.361,00$ & 12.359 & 0,659 \\
\hline Salamina & $19.629,00$ & 12.547 & 0,647 \\
\hline Samaná & $25.220,00$ & 19.110 & 0,614 \\
\hline San José & $7.589,00$ & $*$ & $*$ \\
\hline Supia & $24.931,00$ & 12.162 & 0,651 \\
\hline Victoria & $8.969,00$ & 4.054 & 0,648 \\
\hline Villamaría & $48.041,00$ & 27.590 & 0,706 \\
\hline Viterbo & $12.995,00$ & 11.841 & 0,664 \\
\hline Caldas & $973.226,00$ & 1.206 .653 & 0,758 \\
\hline
\end{tabular}

\begin{tabular}{|c|c|c|c|c|c|}
\hline \multirow[b]{2}{*}{ 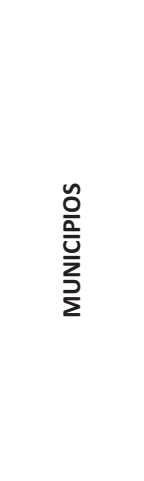 } & \multicolumn{5}{|c|}{ ODM1. ERRADICAR LA POBREZA EXTREMA Y EL HAMBRE } \\
\hline & 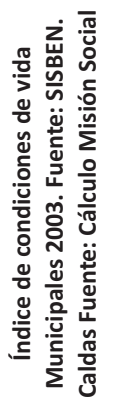 & 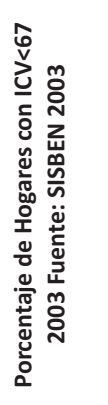 & 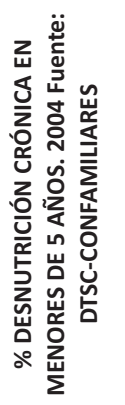 & 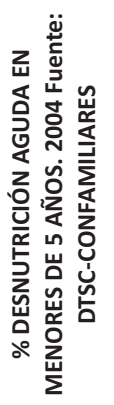 & 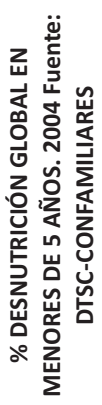 \\
\hline Manizales & 81,59 & $14,4 \%$ & $13,3 \%$ & $1,8 \%$ & $6,9 \%$ \\
\hline Aguadas & 63,74 & $53,7 \%$ & $20,4 \%$ & $5,4 \%$ & $9,9 \%$ \\
\hline Anserma & 64,17 & $48,6 \%$ & $20,2 \%$ & $2,7 \%$ & $10,8 \%$ \\
\hline Aranzazu & $63,33 \%$ & $51,7 \%$ & $20,9 \%$ & $1,9 \%$ & $6,0 \%$ \\
\hline Belalcázar & 58,93 & $60,8 \%$ & $34,6 \%$ & $3,7 \%$ & $20,7 \%$ \\
\hline Chinchiná & 73,68 & $19,7 \%$ & $14,3 \%$ & $2,0 \%$ & $4,3 \%$ \\
\hline Filadelfia & 61,73 & $59,1 \%$ & $15,0 \%$ & $2,4 \%$ & $6,9 \%$ \\
\hline La Dorada & 76,73 & $14,1 \%$ & $13,4 \%$ & $2,5 \%$ & $5,8 \%$ \\
\hline La Meced & 62,91 & $56,8 \%$ & $19,6 \%$ & $3,8 \%$ & $7,2 \%$ \\
\hline Manzanares & 63,92 & $51,5 \%$ & $15,9 \%$ & $2,4 \%$ & $8,8 \%$ \\
\hline Marmato & 64,56 & $47,2 \%$ & $20,8 \%$ & $3,9 \%$ & $7,4 \%$ \\
\hline Marquetalia & 60,84 & $55,0 \%$ & $24,9 \%$ & $2,8 \%$ & $11,8 \%$ \\
\hline Marulanda & 58,27 & $68,8 \%$ & $27,9 \%$ & $3,1 \%$ & $13,5 \%$ \\
\hline Neira & 63,29 & $49,3 \%$ & $23,1 \%$ & $3,5 \%$ & $9,5 \%$ \\
\hline Norcasia & 63,36 & $44,5 \%$ & $13,8 \%$ & $3,7 \%$ & $8,2 \%$ \\
\hline Pácora & 64,18 & $49,9 \%$ & $15,1 \%$ & $1,7 \%$ & $2,5 \%$ \\
\hline Palestina & 68,31 & $32,1 \%$ & $16,5 \%$ & $1,3 \%$ & $6,6 \%$ \\
\hline Pensilvania & 56,23 & $70,5 \%$ & $14,9 \%$ & $4,4 \%$ & $8,2 \%$ \\
\hline Riosucio & 80,92 & $14,4 \%$ & $19,0 \%$ & $3,4 \%$ & $9,0 \%$ \\
\hline Risaralda & 62,07 & $52,6 \%$ & $19,9 \%$ & $2,4 \%$ & $6,9 \%$ \\
\hline Salamina & 66,20 & $47,9 \%$ & $15,8 \%$ & $2,1 \%$ & $6,0 \%$ \\
\hline Samaná & 53,73 & $69,5 \%$ & $21,5 \%$ & $4,0 \%$ & $5,5 \%$ \\
\hline San José & 57,49 & $68,1 \%$ & $35,3 \%$ & $5,2 \%$ & $20,1 \%$ \\
\hline Supia & 67,97 & $45,3 \%$ & $21,9 \%$ & $6,0 \%$ & $7,9 \%$ \\
\hline Victoria & 61,97 & $55,5 \%$ & $7,6 \%$ & $2,4 \%$ & $10,2 \%$ \\
\hline Villamaría & 75,14 & $25,9 \%$ & $10,9 \%$ & $1,5 \%$ & $4,6 \%$ \\
\hline Viterbo & 76,31 & $16,9 \%$ & $17,2 \%$ & $3,5 \%$ & $8,6 \%$ \\
\hline Caldas & 78,35 & & $16,5 \%$ & $2,6 \%$ & $7,4 \%$ \\
\hline
\end{tabular}

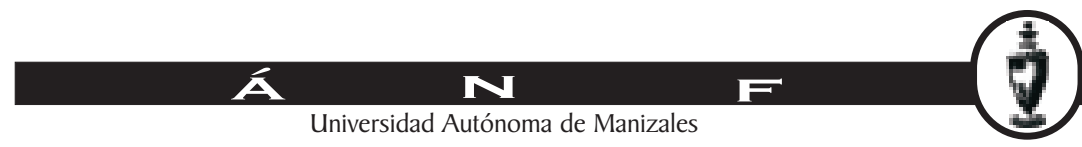




\begin{tabular}{|c|c|c|c|c|c|c|c|c|c|c|}
\hline \multirow[b]{2}{*}{$\begin{array}{l}\frac{\tilde{O}}{a} \\
\frac{\mathrm{U}}{z} \\
\frac{\mathrm{J}}{\Sigma}\end{array}$} & \multicolumn{10}{|c|}{ ODM2. LOGRAR LA PRIMARIA BÁSICA UNIVERSAL } \\
\hline & 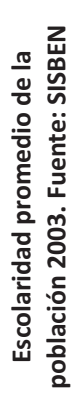 & 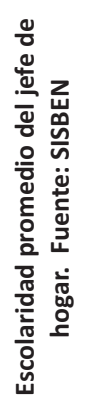 & 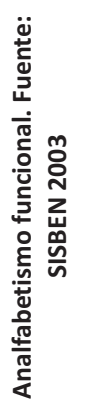 & 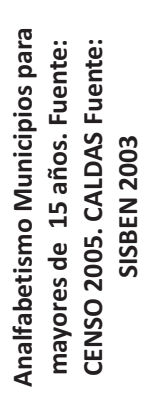 & 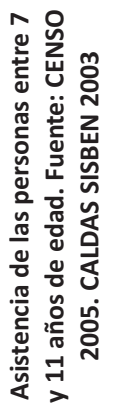 & 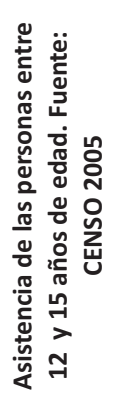 & 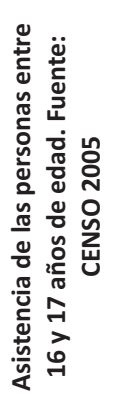 & 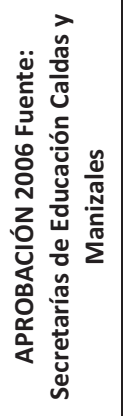 & 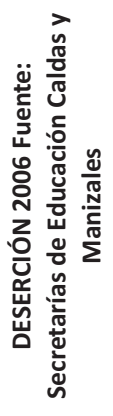 & 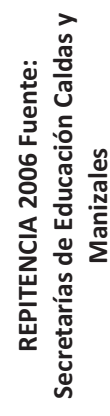 \\
\hline Manizales & 5,12 & 5,56 & $17,3 \%$ & $3,3 \%$ & $94,1 \%$ & $89,6 \%$ & $72,8 \%$ & SD & SD & SD \\
\hline Aguadas & 3,87 & 3,85 & $32,4 \%$ & $12,4 \%$ & $91,2 \%$ & $73,7 \%$ & $45,6 \%$ & $81,30 \%$ & $6,40 \%$ & $11,24 \%$ \\
\hline Anserma & 4,11 & 4,50 & $25,6 \%$ & $8,6 \%$ & $89,4 \%$ & $76,5 \%$ & $51,3 \%$ & $81,96 \%$ & $4,39 \%$ & $11,75 \%$ \\
\hline Aránzazu & 4,02 & 4,26 & $28,2 \%$ & $9,2 \%$ & $93,7 \%$ & $77,5 \%$ & $50,6 \%$ & $79,30 \%$ & $5,84 \%$ & $13,47 \%$ \\
\hline Belalcázar & 3,63 & 3,88 & $32,6 \%$ & $16,1 \%$ & $90,6 \%$ & $74,1 \%$ & $40,3 \%$ & $78,00 \%$ & $6,50 \%$ & $14,82 \%$ \\
\hline Chinchiná & 4,00 & 4,21 & $26,6 \%$ & $7,0 \%$ & $90,6 \%$ & $82,3 \%$ & $62,5 \%$ & $80,02 \%$ & $3,56 \%$ & $15,44 \%$ \\
\hline Filadelfia & 3,91 & 4,12 & $29,8 \%$ & $10,0 \%$ & $89,9 \%$ & $68,2 \%$ & $53,7 \%$ & $81,03 \%$ & $6,51 \%$ & $10,84 \%$ \\
\hline La Dorada & 4,47 & 4,99 & $21,3 \%$ & $9,5 \%$ & $87,7 \%$ & $81,8 \%$ & $60,4 \%$ & $74,31 \%$ & $7,72 \%$ & 13,89 \\
\hline La Meced & 3,82 & 3,85 & $32,8 \%$ & $12,3 \%$ & $94,5 \%$ & $83,2 \%$ & $45,9 \%$ & $76,00 \%$ & $8,62 \%$ & $13.06 \%$ \\
\hline Manzanares & 3,77 & 4,16 & $29,5 \%$ & $12,3 \%$ & $93,9 \%$ & $76,8 \%$ & $46,1 \%$ & $84,79 \%$ & $3,65 \%$ & $11,14 \%$ \\
\hline Marmato & 4,13 & 4,49 & $23,3 \%$ & $9,0 \%$ & $95,6 \%$ & $80,3 \%$ & $55,5 \%$ & $75,27 \%$ & $9,70 \%$ & $14,25 \%$ \\
\hline Marquetalia & 3,67 & 3,75 & $31,2 \%$ & $12,5 \%$ & $95,9 \%$ & $72,3 \%$ & $40,2 \%$ & $80,07 \%$ & $5,67 \%$ & $13,52 \%$ \\
\hline Marulanda & 3,46 & 6,94 & $33,3 \%$ & $10,9 \%$ & $92,5 \%$ & $77,9 \%$ & $33,6 \%$ & $83,63 \%$ & $5,87 \%$ & $10,35 \%$ \\
\hline Neira & 4,02 & 4,05 & $29,4 \%$ & $13,8 \%$ & $88,4 \%$ & $74,4 \%$ & $50,2 \%$ & $83.02 \%$ & $3,87 \%$ & $12,53 \%$ \\
\hline Norcasia & 3,48 & 4,05 & $28,6 \%$ & $14,7 \%$ & $95,8 \%$ & $83,0 \%$ & $49,8 \%$ & $69,60 \%$ & $22,88 \%$ & $14,20 \%$ \\
\hline Pácora & 3,89 & 3,93 & $32,7 \%$ & $11,2 \%$ & $93,6 \%$ & $77,9 \%$ & $50,3 \%$ & $87,02 \%$ & $3,90 \%$ & $8,24 \%$ \\
\hline Palestina & 3,95 & 4,08 & $27,5 \%$ & $11,6 \%$ & $88,8 \%$ & $78,0 \%$ & $58,5 \%$ & $75,53 \%$ & $6,45 \%$ & $17,17 \%$ \\
\hline Pensilvania & 3,53 & 3,69 & $31,4 \%$ & $13,0 \%$ & $89,0 \%$ & $69,6 \%$ & $46,4 \%$ & $76,20 \%$ & $11.10 \%$ & $11,60 \%$ \\
\hline Riosucio & 6,02 & 6,51 & $15,0 \%$ & $6,9 \%$ & $86,4 \%$ & $74,9 \%$ & $57,0 \%$ & $83,62 \%$ & $7,07 \%$ & $8,73 \%$ \\
\hline Risaralda & 3,60 & 3,81 & $30,8 \%$ & $12,5 \%$ & $93,5 \%$ & $80,8 \%$ & $45,1 \%$ & $71,25 \%$ & $4,98 \%$ & $22,45 \%$ \\
\hline Salamina & 4,26 & 4,62 & $27,8 \%$ & $10,0 \%$ & $91,6 \%$ & $79,6 \%$ & $60,5 \%$ & $76,70 \%$ & $12,40 \%$ & $10,20 \%$ \\
\hline Samaná & 3,07 & 3,19 & $35,9 \%$ & $13,9 \%$ & $93,2 \%$ & $67,7 \%$ & $32,6 \%$ & $68,60 \%$ & $9,82 \%$ & $20,67 \%$ \\
\hline San José & 3,57 & 3,72 & $32,1 \%$ & $19,0 \%$ & $88,9 \%$ & $75,7 \%$ & $43,1 \%$ & $88,47 \%$ & $6,02 \%$ & $5,17 \%$ \\
\hline Supia & 4,35 & 4,68 & $24,6 \%$ & $8,8 \%$ & $94,1 \%$ & $81,8 \%$ & $51,3 \%$ & $85,14 \%$ & $6,97 \%$ & $7,58 \%$ \\
\hline Victoria & 3,69 & 3,99 & $29,4 \%$ & $14,4 \%$ & $92,2 \%$ & $81,7 \%$ & $51,1 \%$ & $75,11 \%$ & $5,05 \%$ & $19,09 \%$ \\
\hline Villamaría & 4,93 & 5,34 & $20,0 \%$ & $7,0 \%$ & $90,2 \%$ & $84,8 \%$ & $69,0 \%$ & $87,31 \%$ & $4,38 \%$ & $7,99 \%$ \\
\hline Viterbo & 4,50 & 4,90 & $22,8 \%$ & $8,8 \%$ & $97,0 \%$ & $89,1 \%$ & $62,9 \%$ & $85,69 \%$ & $3,37 \%$ & $10,12 \%$ \\
\hline Caldas & 4,59 & & & $8,8 \%$ & $95,8 \%$ & & & & & \\
\hline
\end{tabular}

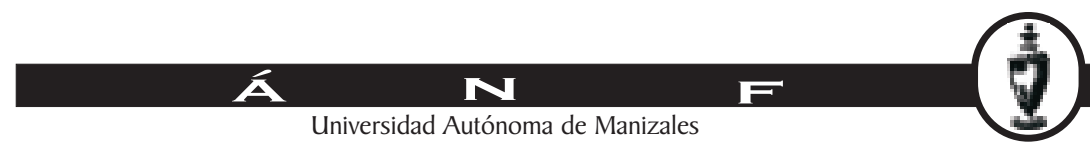




\begin{tabular}{|c|c|c|}
\hline \multirow[b]{2}{*}{ 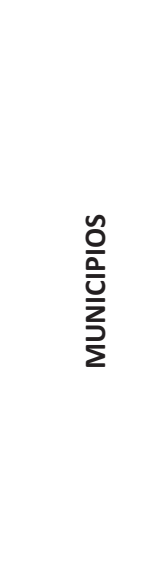 } & \multicolumn{2}{|c|}{$\begin{array}{l}\text { ODM 3. PROMOVER LA } \\
\text { EQUIDAD DE GÉNERO Y LA } \\
\text { AUTONOMÍA DE LA MUJER }\end{array}$} \\
\hline & 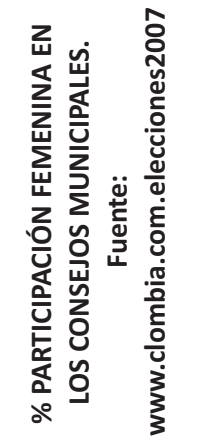 & 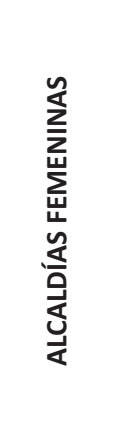 \\
\hline Manizales & $21,05 \%$ & $0,00 \%$ \\
\hline Aguadas & $0,00 \%$ & $0,00 \%$ \\
\hline Anserma & $0,00 \%$ & $0,00 \%$ \\
\hline Aranzazu & $27,27 \%$ & $0,00 \%$ \\
\hline Belalcázar & $9,09 \%$ & $0,00 \%$ \\
\hline Chinchiná & $13,33 \%$ & $100,00 \%$ \\
\hline Filadelfia & $18,18 \%$ & $0,00 \%$ \\
\hline La Dorada & $26,67 \%$ & $0,00 \%$ \\
\hline La Meced & $22,22 \%$ & $0,00 \%$ \\
\hline Manzanares & $15,38 \%$ & $0,00 \%$ \\
\hline Marmato & $0,00 \%$ & $0,00 \%$ \\
\hline Marquetalia & $9,09 \%$ & $0,00 \%$ \\
\hline Marulanda & $0,00 \%$ & $0,00 \%$ \\
\hline Neira & $23,08 \%$ & $0,00 \%$ \\
\hline Norcasia & $22,22 \%$ & $0,00 \%$ \\
\hline Pacora & $18,18 \%$ & $100,00 \%$ \\
\hline Palestina & $9,09 \%$ & $0,00 \%$ \\
\hline Pensilvania & $15,38 \%$ & $0,00 \%$ \\
\hline Riosucio & $20,00 \%$ & $0,00 \%$ \\
\hline Risaralda & $18,18 \%$ & $0,00 \%$ \\
\hline Salamina & $23,08 \%$ & $0,00 \%$ \\
\hline Samaná & $7,69 \%$ & $0,00 \%$ \\
\hline San José & $0,00 \%$ & $0,00 \%$ \\
\hline Supia & $15,38 \%$ & $0,00 \%$ \\
\hline Victoria & $22,22 \%$ & $0,00 \%$ \\
\hline Villamaría & $15,38 \%$ & $0,00 \%$ \\
\hline Viterbo & $0,00 \%$ & $0,00 \%$ \\
\hline Caldas & $14,29 \%$ & $7,40 \%$ \\
\hline
\end{tabular}

\begin{tabular}{|c|c|c|c|}
\hline \multirow[b]{2}{*}{$\begin{array}{l}\text { 음 } \\
\frac{0}{\underline{U}} \\
\frac{2}{\Sigma}\end{array}$} & \multicolumn{3}{|c|}{$\begin{array}{l}\text { ODM 4. REDUCIR LA MORTALIDAD } \\
\text { INFANTIL }\end{array}$} \\
\hline & 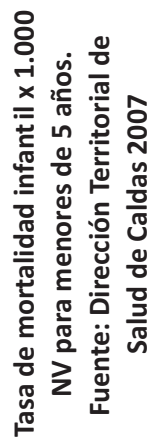 & 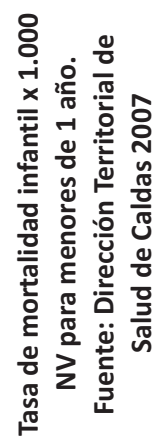 & 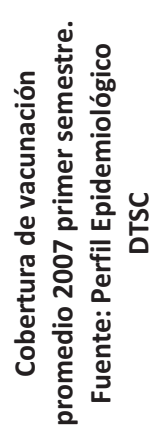 \\
\hline Manizales & 16,65 & 14,06 & $103,6 \%$ \\
\hline Aguadas & 13,85 & 13,85 & $89,5 \%$ \\
\hline Anserma & 14,14 & 12,12 & $86,6 \%$ \\
\hline Aranzazu & 10,99 & 10,99 & $102,4 \%$ \\
\hline Belalcázar & 23,12 & 23,12 & $95,9 \%$ \\
\hline Chinchiná & 20,30 & 15,23 & $101,3 \%$ \\
\hline Filadelfia & 26,14 & 26,14 & $88,7 \%$ \\
\hline La Dorada & 16,79 & 10,50 & $95,1 \%$ \\
\hline La Meced & 24,10 & 24,10 & $90,2 \%$ \\
\hline Manzanares & 13,94 & 10,45 & $94,8 \%$ \\
\hline Marmato & 40,32 & 24,29 & $101,3 \%$ \\
\hline Marquetalia & 13,82 & 9,22 & $98,4 \%$ \\
\hline Marulanda & 64,52 & 64,52 & $90,6 \%$ \\
\hline Neira & 18,13 & 15,54 & $112,9 \%$ \\
\hline Norcasia & 6,90 & 6,90 & $133,7 \%$ \\
\hline Pacora & 36,46 & 36,46 & $93,9 \%$ \\
\hline Palestina & 20,76 & 20,76 & $93,5 \%$ \\
\hline Pensilvania & 8,26 & 8,26 & $90,7 \%$ \\
\hline Riosucio & 16,33 & 10,88 & $116,8 \%$ \\
\hline Risaralda & 24,27 & 19,42 & $94,7 \%$ \\
\hline Salamina & 16,00 & 8,00 & $108,9 \%$ \\
\hline Samaná & 21,88 & 21,88 & $92,2 \%$ \\
\hline San José & 0,00 & SD & $131,5 \%$ \\
\hline Supia & 19,29 & 16,08 & $95,0 \%$ \\
\hline Victoria & 0,00 & SD & $107,5 \%$ \\
\hline Villamaría & 10,19 & 10,19 & $128,5 \%$ \\
\hline Viterbo & 14,85 & 14,85 & $95,9 \%$ \\
\hline Caldas & & & \\
\hline
\end{tabular}

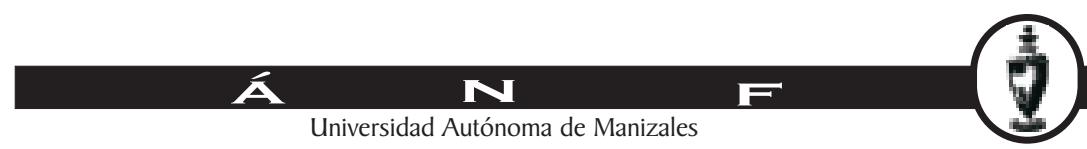




\begin{tabular}{|c|c|c|c|c|c|c|}
\hline \multirow[b]{2}{*}{$\begin{array}{l}\text { 음 } \\
\frac{0}{2} \\
\frac{2}{2}\end{array}$} & \multicolumn{6}{|c|}{ ODM 5. MEJORAR LA SALUD SEXUAL Y REPRODUCTIVA } \\
\hline & 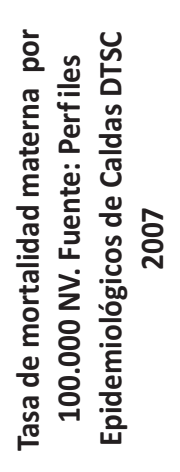 & 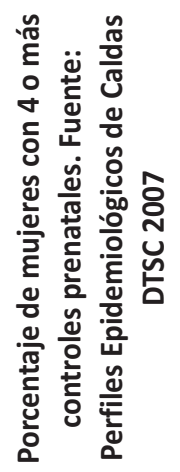 & 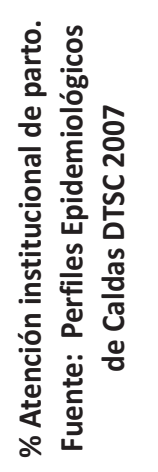 & 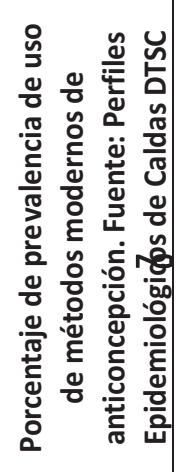 & 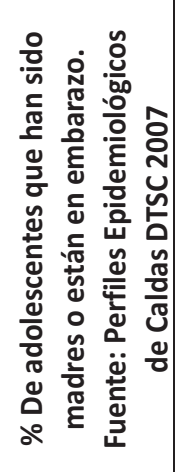 & 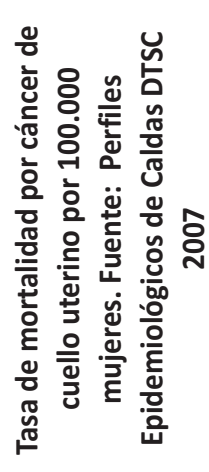 \\
\hline Manizales & 86,51 & 90,50 & 99,60 & 76,10 & 23,20 & 8 \\
\hline Aguadas & 277,01 & 90,00 & 97,00 & 71,00 & 22,00 & 0,00 \\
\hline Anserma & 0,00 & 89,00 & 98,00 & 76,00 & 27,00 & 12,00 \\
\hline Aranzazu & 0,00 & 95,00 & 98,00 & 65,00 & 222,00 & 32,00 \\
\hline Belalcázar & 0,00 & 87,90 & 96,00 & 59,00 & 26,00 & 0,00 \\
\hline Chinchiná & 0,00 & 85,00 & 99,00 & 75,50 & 27,00 & 0,00 \\
\hline Filadelfia & 0,00 & 84,00 & 100,00 & 58,00 & 23,00 & 0,00 \\
\hline La Dorada & 69,98 & 90,00 & 94,00 & 53,50 & 29,00 & 25,00 \\
\hline La Meced & 0,00 & 92,80 & 98,30 & 64,00 & 29,00 & 32,00 \\
\hline Manzanares & 696,86 & 95,00 & 90,00 & 68,00 & 29,00 & 0,00 \\
\hline Marmato & 0,00 & 100,00 & 90,00 & 69,00 & 23,00 & 25,00 \\
\hline Marquetalia & 0,00 & 71,10 & 95,50 & 62,00 & 26,00 & 0,00 \\
\hline Marulanda & 0,00 & 61,10 & 85,00 & 52,00 & 19,00 & 0,00 \\
\hline Neira & 0,00 & 100,00 & 90,00 & 57,00 & 28,00 & 7,00 \\
\hline Norcasia & 0,00 & 87,00 & 93,00 & 58,00 & 26,00 & 31,00 \\
\hline Pacora & 0,00 & 83,40 & 98,50 & 62,00 & 28,00 & 28,00 \\
\hline Palestina & 0,00 & 95,00 & 100,00 & 56,00 & 30,00 & 12,00 \\
\hline Pensilvania & 0,00 & 68,00 & 97,00 & 53,00 & 26,00 & 9,00 \\
\hline Riosucio & 0,00 & 98,00 & 99,00 & 52,00 & 27,00 & 0,00 \\
\hline Risaralda & 0,00 & 95,00 & 100,00 & 64,00 & 29,00 & 20,00 \\
\hline Salamina & 0,00 & 92,00 & 100,00 & 62,00 & 28,00 & 0,00 \\
\hline Samaná & 0,00 & 90,00 & 95,00 & 58,00 & 27,00 & 45,00 \\
\hline San José & 0,00 & 100,00 & 100,00 & 64,00 & 28,00 & 0,00 \\
\hline Supia & 0,00 & 90,00 & 90,00 & 68,00 & 28,00 & 8,00 \\
\hline Victoria & 0,00 & 90,00 & 94,00 & 69,00 & 32,00 & 0,00 \\
\hline Villamaría & 169,78 & 88,00 & 97,00 & 75,40 & 26,00 & 4,00 \\
\hline Viterbo & 0,00 & 90,00 & 98,00 & 59,00 & 26,00 & 0,00 \\
\hline Caldas & 68,58 & 87,50 & 96,00 & 63,20 & 26,50 & 9,00 \\
\hline
\end{tabular}

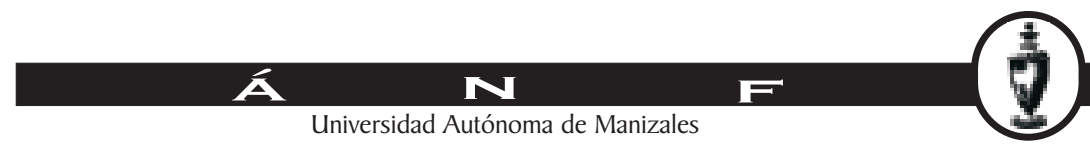




\begin{tabular}{|c|c|c|c|c|c|}
\hline \multirow[b]{2}{*}{$\begin{array}{l}\frac{\text { 음 }}{\text { 은 }} \\
\frac{2}{2}\end{array}$} & \multicolumn{5}{|c|}{ ODM 6. COMBATIR EL VIH SIDA, LA MALRIA Y EL DENGUE } \\
\hline & 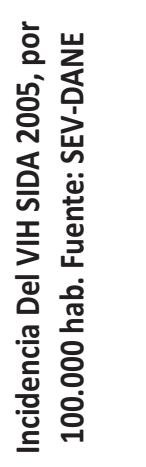 & 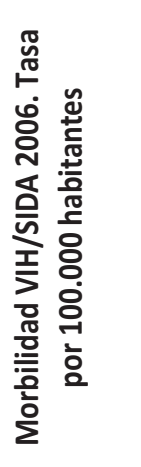 & 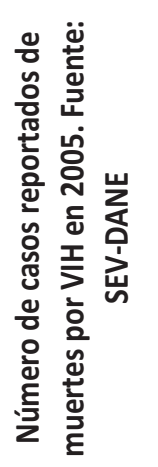 & 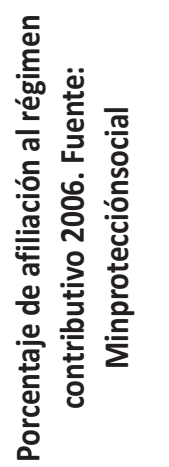 & 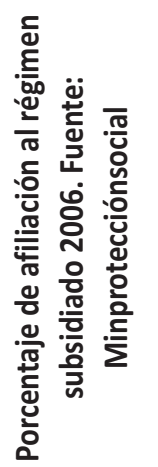 \\
\hline Manizales & 8,96 & 23,64 & 33 & $68,6 \%$ & $31,4 \%$ \\
\hline Aguadas & SD & SD & SD & $10,3 \%$ & $89,7 \%$ \\
\hline Anserma & SD & 2,99 & SD & $16,4 \%$ & $83,6 \%$ \\
\hline Aranzazu & 8,21 & 16,46 & 1 & $14,3 \%$ & $85,7 \%$ \\
\hline Belalcázar & SD & 17,70 & SD & $10,9 \%$ & $89,1 \%$ \\
\hline Chinchiná & 13,64 & 23,52 & 7 & $32,9 \%$ & $67,1 \%$ \\
\hline Filadelfia & 16,35 & 8,19 & 2 & $8,0 \%$ & $92,0 \%$ \\
\hline La Dorada & 7,09 & 18,89 & 5 & $37,6 \%$ & $62,4 \%$ \\
\hline La Meced & SD & SD & SD & $13,0 \%$ & $87,0 \%$ \\
\hline Manzanares & SD & SD & SD & $10,2 \%$ & $89,8 \%$ \\
\hline Marmato & SD & SD & SD & $24,0 \%$ & $76,0 \%$ \\
\hline Marquetalia & 7,20 & SD & 1 & $7,3 \%$ & $92,7 \%$ \\
\hline Marulanda & SD & SD & SD & $8,1 \%$ & $91,9 \%$ \\
\hline Neira & 3,67 & 7,42 & 1 & $14,1 \%$ & $85,9 \%$ \\
\hline Norcasia & SD & 15,68 & SD & $1,2 \%$ & $98,8 \%$ \\
\hline Pacora & SD & 13,94 & SD & $12,0 \%$ & $88,0 \%$ \\
\hline Palestina & SD & SD & SD & $12,9 \%$ & $87,1 \%$ \\
\hline Pensilvania & SD & SD & SD & $13,0 \%$ & $87,0 \%$ \\
\hline Riosucio & 2,79 & 2,80 & 1 & $10,9 \%$ & $89,1 \%$ \\
\hline Risaralda & SD & 29,53 & SD & $6,2 \%$ & $93,8 \%$ \\
\hline Salamina & 5,46 & 16,57 & 1 & $15,4 \%$ & $84,6 \%$ \\
\hline Samaná & 4,07 & SD & 1 & $3,8 \%$ & $96,2 \%$ \\
\hline San José & SD & SD & SD & SD & SD \\
\hline Supia & SD & 8,33 & SD & $14,5 \%$ & $85,5 \%$ \\
\hline Victoria & SD & SD & SD & $7,8 \%$ & $92,2 \%$ \\
\hline Villamaría & 8,88 & 17,87 & 4 & $31,2 \%$ & $68,8 \%$ \\
\hline Viterbo & SD & SD & SD & $32,1 \%$ & $97,9 \%$ \\
\hline Caldas & & & & & \\
\hline
\end{tabular}

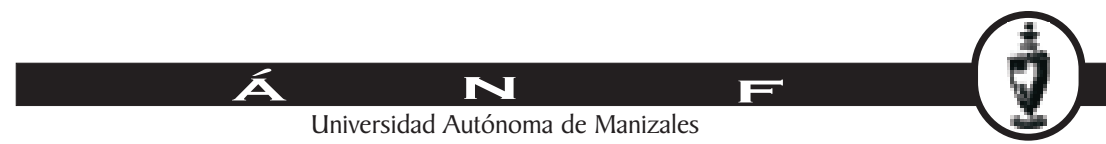




\begin{tabular}{|c|c|c|c|c|c|c|c|c|c|c|}
\hline \multirow[b]{2}{*}{$\begin{array}{l}\frac{\tilde{0}}{\frac{0}{2}} \\
\frac{\mathrm{v}}{z} \\
\frac{1}{\Sigma}\end{array}$} & \multicolumn{10}{|c|}{ ODM 7. GARANTIZAR LA SOSTENIBILIDAD AMBIENTAL } \\
\hline & 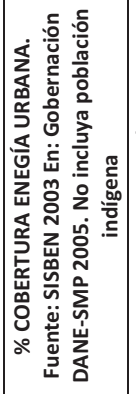 & 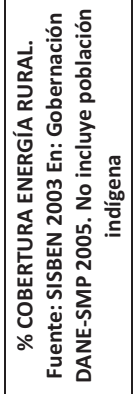 & 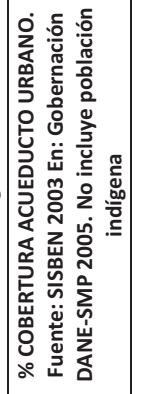 & 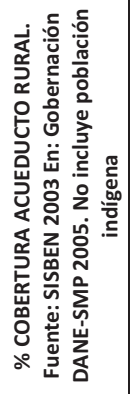 & 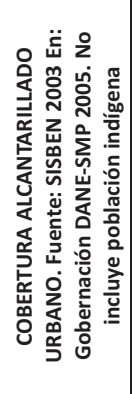 & 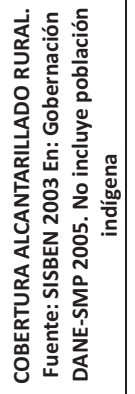 & 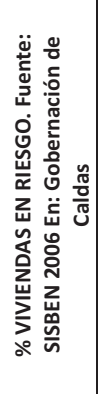 & 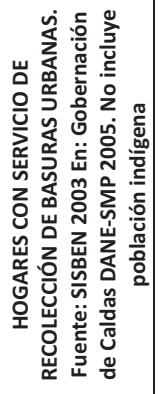 & 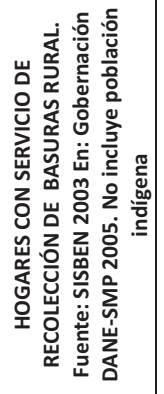 & 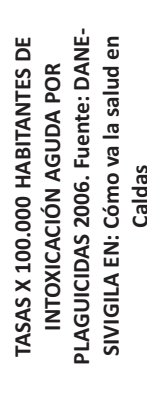 \\
\hline Manizales & 98,80 & 97,00 & 99,10 & 71,10 & 98,60 & 41,30 & 10,70 & 99,60 & 54,20 & 84,11 \\
\hline Aguadas & 97,70 & 92,20 & 98,40 & 65,00 & 97,70 & 15,50 & 2,10 & 95,20 & 11,30 & 49,36 \\
\hline Anserma & 99,10 & 95,10 & 99,10 & 1,90 & 99,00 & 2,30 & 4,00 & 99,40 & 4,80 & 17,09 \\
\hline Aránzazu & 98,20 & 97,00 & 98,20 & 37,20 & 97,90 & 1,60 & 1,40 & 98,60 & 0,80 & 31,21 \\
\hline Belalcazar & 99,20 & 97,10 & 97,50 & 49,50 & 93,30 & 7,30 & 1,00 & 96,70 & 8,10 & 101,07 \\
\hline Chinchiná & 99,80 & 97,80 & 99,10 & 69,50 & 98,50 & 35,10 & 5,50 & 99,10 & 29,20 & 84,11 \\
\hline Filadelfia & 99,30 & 97,60 & 98,10 & 53,60 & 98,00 & 22,20 & 0,70 & 98,00 & 15,80 & 7,85 \\
\hline La Dorada & 99,10 & 79,10 & 98,40 & 54,20 & 97,90 & 32,60 & 23,20 & 98,60 & 35,70 & 1,37 \\
\hline La Meced & 99,20 & 95,10 & 99,40 & 66,60 & 96,10 & 1,10 & 0,40 & 96,20 & 14,90 & 14,81 \\
\hline Manzanares & 99,30 & 93,80 & 95,30 & 82,30 & 86,30 & 9,20 & 4,70 & 92,00 & 6,20 & 27,88 \\
\hline Marmato & 100,00 & 96,50 & 92,10 & 85,20 & 67,70 & 36,80 & 3,00 & 75,60 & 26,30 & 11,82 \\
\hline Marquetalia & 99,00 & 93,50 & 98,50 & 34,50 & 91,00 & 1,70 & 1,30 & 92,10 & 1,50 & 54,06 \\
\hline Marulanda & 99,20 & 83,80 & 98,10 & 37,70 & 95,50 & 29,80 & 2,00 & 96,20 & 27,40 & SD \\
\hline Neira & 99,70 & 96,80 & 98,80 & 59,00 & 98,00 & 14,10 & 1,10 & 97,00 & 5,60 & 42,64 \\
\hline Norcasia & 98,90 & 46,20 & 97,80 & 13,80 & 89,80 & 3,60 & 2,80 & 98,20 & 5,40 & 28,97 \\
\hline Pacora & 99,30 & 96,40 & 99,20 & 52,30 & 94,30 & 3,50 & 1,30 & 98,80 & 3,20 & 26,32 \\
\hline Palestina & 98,60 & 98,20 & 97,10 & 64,20 & 95,10 & 16,30 & 3,60 & 97,10 & 29,10 & 121,97 \\
\hline Pensilvania & 99,10 & 95,50 & 97,40 & 34,10 & 93,60 & 20,80 & 4,60 & 98,00 & 9,90 & 41,62 \\
\hline Riosucio & 98,90 & 94,80 & 98,00 & 88,00 & 97,20 & 61,20 & 1,60 & 92,80 & 21,60 & 5,50 \\
\hline Risaralda & 99,60 & 96,70 & 99,20 & 47,80 & 97,40 & 6,70 & 0,80 & 98,90 & 14,20 & 74,91 \\
\hline Salamina & 98,70 & 94,10 & 97,10 & 61,00 & 97,20 & 4,30 & 3,10 & 95,40 & 15,40 & 34,50 \\
\hline Samana & 98,70 & 79,50 & 97,80 & 37,60 & 88,10 & 12,10 & 9,50 & 97,00 & 11,30 & 27,29 \\
\hline San Jose & 99,70 & 97,70 & 96,40 & 40,10 & 90,80 & 7,90 & 0,40 & 93,60 & 4,40 & 105,65 \\
\hline Supia & 99,50 & 92,40 & 99,50 & 69,00 & 91,30 & 6,60 & 4,70 & 99,10 & 4,90 & 12,07 \\
\hline Victoria & 97,30 & 77,70 & 94,30 & 54,20 & 85,60 & 10,50 & 1,10 & 91,00 & 0,90 & 32,73 \\
\hline Villamaría & 99,40 & 94,30 & 98,30 & 59,40 & 96,20 & 34,20 & 4,20 & 96,70 & 30,30 & 28,06 \\
\hline Viterbo & 99,50 & 97,50 & 98,90 & 61,20 & 98,40 & 27,80 & 1,40 & 99,20 & 5,40 & 136,78 \\
\hline Caldas & 99,30 & 93,00 & 98,50 & 52,70 & 96,80 & 16,70 & 3,70 & 98,00 & 16,10 & 25,29 \\
\hline
\end{tabular}

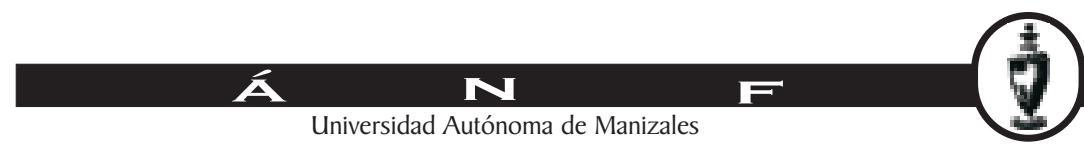




\begin{tabular}{|c|c|c|c|c|c|c|c|c|c|c|c|}
\hline \multirow[b]{2}{*}{ 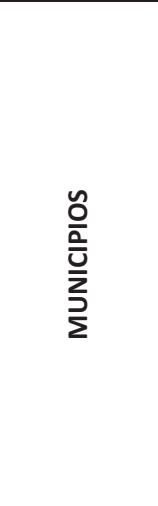 } & \multicolumn{11}{|c|}{ ODM 8. FOMENTAR UNA ASOCIACIÓN MUNDIAL PARA EL DESARROLLO } \\
\hline & 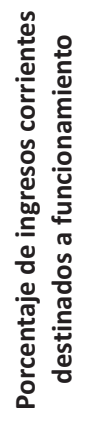 & 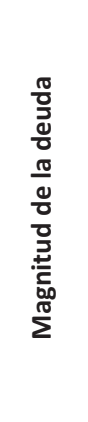 & 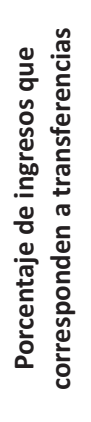 & 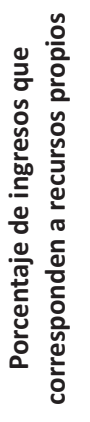 & 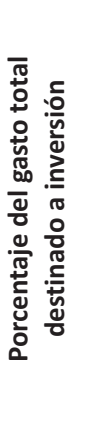 & 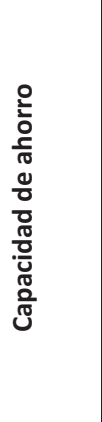 & 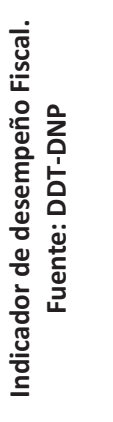 & 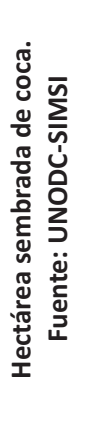 & 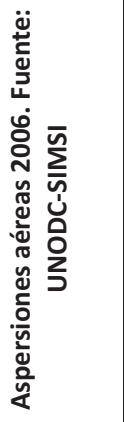 & 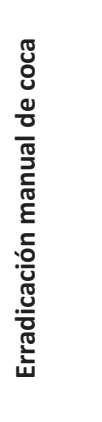 & 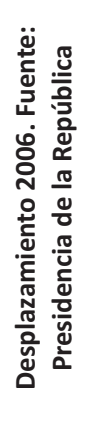 \\
\hline Manizales & 61,33 & 40,50 & 60,26 & 29,66 & 74,80 & 30,70 & 56,52 & SD & SD & SD & 113 \\
\hline Aguadas & 84,83 & 3,06 & 83,75 & 8,00 & 81,64 & 13,17 & 53,47 & SD & SD & SD & 63 \\
\hline Anserma & 75,82 & 0,00 & 72,46 & 21,67 & 69,44 & 20,30 & 58,16 & SD & SD & SD & 173 \\
\hline Aranzazu & 62,62 & 2,17 & 78,08 & 11,50 & 78,18 & 15,12 & 55,87 & SD & SD & SD & 26 \\
\hline Belalcázar & 80,63 & 12,23 & 74,15 & 15,49 & 77,42 & 18,63 & 55,59 & SD & SD & SD & 3 \\
\hline Chinchiná & 94,10 & 47,40 & 53,95 & 39,05 & 49,61 & 3,16 & 46,24 & SD & SD & SD & 54 \\
\hline Filadelfia & 63,84 & 8,40 & 82,58 & 8,79 & 81,01 & 14,03 & 53,88 & SD & SD & SD & 20 \\
\hline La Dorada & 59,01 & 15,52 & 53,58 & 33,89 & 69,53 & 44,30 & 64,07 & SD & SD & SD & 83 \\
\hline La Meced & 52,47 & 0,72 & 82,63 & 9,73 & 83,25 & 29,37 & 58,20 & SD & SD & SD & 8 \\
\hline Manzanares & 75,55 & 6,10 & 80,32 & 9,48 & 79,25 & 23,22 & 55,91 & SD & SD & SD & 50 \\
\hline Marmato & 82,04 & 0,80 & 46,12 & 2,34 & 86,10 & $-0,66$ & 58,19 & SD & SD & SD & SD \\
\hline Marquetalia & 70,89 & 9,87 & 77,18 & 10,23 & 81,60 & 13,70 & 54,74 & SD & SD & SD & 72 \\
\hline Marulanda & 61,06 & 0,00 & 73,62 & 9,82 & 78,29 & 16,43 & 56,91 & SD & SD & SD & 22 \\
\hline Neira & 100,06 & 7,01 & 70,98 & 21,96 & 69,99 & 3,59 & 50,33 & SD & SD & SD & 59 \\
\hline Norcasia & 49,55 & 0,00 & 76,94 & 13,74 & 81,27 & 38,55 & 60,98 & 46 & 328,53 & 208,87 & 47 \\
\hline Pácora & 56,90 & 19,25 & 78,61 & 10,45 & 81,28 & 10,47 & 52,29 & SD & SD & SD & 11 \\
\hline Palestina & 53,40 & 11,10 & 66,76 & 24,24 & 76,41 & 35,91 & 61,06 & SD & SD & SD & 12 \\
\hline Pensilvania & 66,74 & 3,85 & 79,63 & 11,25 & 83,57 & 23,99 & 57,56 & 7 & 39,08 & 48,97 & 149 \\
\hline Riosucio & 64,00 & 0,33 & 71,88 & 17,54 & 76,66 & 33,59 & 60,80 & SD & SD & SD & 138 \\
\hline Risaralda & 53,43 & 0,00 & 75,55 & 14,24 & 98,76 & 28,50 & 62,65 & SD & SD & SD & 9 \\
\hline Salamina & 86,58 & 93,31 & 80,62 & 12,49 & 70,93 & 13,87 & 36,16 & SD & SD & SD & 50 \\
\hline Samaná & 86,27 & 0,35 & 80,44 & 2,48 & 88,56 & 30,68 & 57,17 & 132 & 677,64 & 282,21 & 516 \\
\hline San José & 61,28 & 9,43 & 80,42 & 8,05 & 81,78 & 18,15 & 54,68 & SD & SD & SD & 6 \\
\hline Supia & 61,02 & 13,53 & 72,76 & 17,89 & 79,34 & 35,58 & 59,06 & SD & SD & SD & 50 \\
\hline Victoria & 66,93 & 0,35 & 71,93 & 20,70 & 75,99 & 39,21 & 62,07 & 4 & 23,2 & 11,5 & 18 \\
\hline Villamaría & 50,40 & 22,04 & 57,21 & 31,66 & 74,49 & 55,03 & 64,45 & SD & SD & SD & 17 \\
\hline Viterbo & 69,51 & 24,89 & 60,39 & 15,15 & 70,54 & 31,75 & 56,43 & SD & SD & SD & 13 \\
\hline Caldas & & & & & & & & & & & \\
\hline
\end{tabular}

* Las cifras de Norcasia y San José se encuentran incluidas en Samaná y Risaralda respectivamente SD: Sin Datos

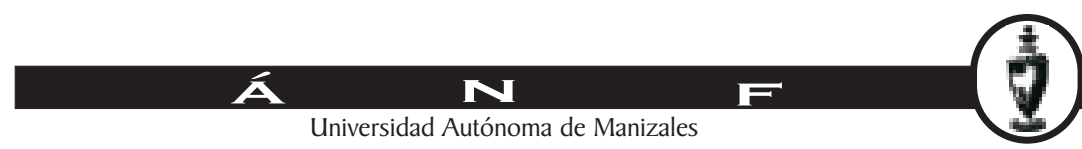




\section{Planes de Desarrollo y ODM}

No se encuentra un ejercicio en el Departamento que haya sustentado la estructura o la construcción de su Plan de Desarrollo sobre los Objetivos del Milenio; hecho que se justifica en primera instancia en la rigidez que perciben y asumen, quienes revisan los planes, en los modelos impuestos por entidades de ámbitos territoriales superiores, donde valdría la pena ahondar, en futuras reflexiones, en la discusión de la dificultad que esto representa para desarrollar las capacidades de autodeterminación del desarrollo de las comunidades, las garantías para el desarrollo endógeno, los principios de la descentralización. Esta rigidez lleva a que se planifique fundamentalmente sobre los recursos del sistema general de participaciones y que los planes de desarrollo se esquematicen en una estructura plana y tradicional de atención de necesidades y metas preestablecidas por sectores; lo cual es necesario pero no necesariamente es todo lo que se necesita.

Una segunda consideración con respecto a los Objetivos del Milenio, es que estos han sido muy bien publicitados en ambientes académicos y algunos institucionales del ámbito nacional, pero no han llegado a las conciencias institucionales locales y comunitarias; éste es un tránsito que está comenzando a suceder y se considera como un hito importante el haber logrado por lo menos la inclusión de los Objetivos en la mayoría de los Planes de Desarrollo como referente de Planificación e incluso, en algunos casos, como parte del análisis de la línea base del municipio.

En un análisis puntual sobre cada uno de los 27 planes de desarrollo municipales se pueden obtener las siguientes conclusiones con respecto a la consideración que se tuvo de los Objetivos del Milenio:

\begin{tabular}{|l|}
\hline AGUADAS 2008 - 2011 Bicentenario con visión de futuro \\
\hline Se estructura como una estrategia de acciones sectoriales conceptualizadas en cinco \\
dimensiones (Capital Humano, Bienestar Social, Desarrollo del Hábitat, Desarrollo Comunitario \\
y Desarrollo Económico) y una estrategia institucional. \\
El objetivo principal es "potenciar las capacidades de los Aguadeños para desarrollar sus \\
proyectos de vida en un ambiente colectivo, solidario y participativo, caracterizado por las \\
condiciones de paz y de dotación de herramientas y mecanismos que garanticen la \\
sostenibilidad y la competitividad". \\
Los ODM tienen una incidencia importante desde su consideración como parte, cada uno de \\
ellos, de la línea base del municipio e inclusión de los mismos en la formulación de metas e \\
indicadores, retomando y ajustando el ejercicio del CEA con las bases de datos locales.
\end{tabular}

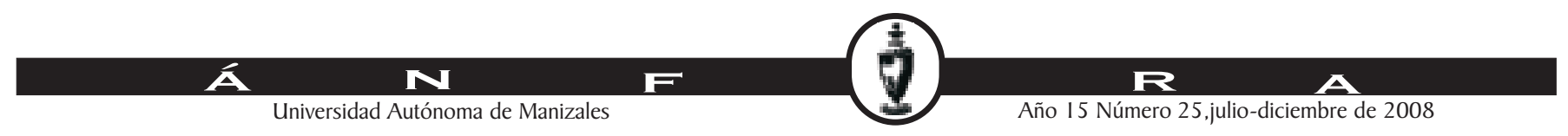


ANSERMA con dignidad y honor

Tiene como objetivo "desarrollar la dignidad y calidad de vida del ser humano, es decir, su bienestar en una sociedad". Estructuralmente lo componen tres dimensiones que apuntan a los tres sujetos básicos del desarrollo: las personas, el territorio que habitan y las instituciones que lo regulan; para lo cual se formulan tres políticas respectivas: La política de desarrollo humano, la política de desarrollo territorial y la política de desarrollo institucional.

Los ODM, según referencia el plan, fueron considerados como un criterio orientador para la definición de metas, sin que éstas se liguen necesariamente a un ejercicio sobre los objetivos.

ARANZAZU 2008 - 2011 Futuro y Desarrollo Social

El Plan de Desarrollo se plantea sobre una estructura tradicional de programas por sectores, agrupados por áreas (Social, Desarrollo Económico, Físico Territorial, Defensa y Seguridad, Desarrollo Institucional). Por cada sector se establecen metas e indicadores, que de alguna manera incluyen algunos que impactan sobre los objetivos del milenio; pero no se hace una referencia exacta a ellos, ni expresa el documento su consideración como referente.

BELALCAZAR 2008 - 2011 Por un Belalcazar para todos

El plan de desarrollo establece programas sobre 6 ejes (Desarrollo de obras públicas y planeación; Integración Social, Desarrollo de agricultura y medio ambiente; Desarrollo educativo; Desarrollo de cultura, turismo y deporte; Desarrollo de Gobierno y convivencia ciudadana). En cada eje define metas de producto y de resultado; que en un ejercicio de revisión detallada permite identificar algunas relacionadas con los objetivos del milenio; sin que el plan haga alusión o descripción directa a ellos, ó los establezca como un referente para la definición del mismo.

CHINCHINA Compromiso de Todos 2008 - 2011

La referencia que se hace a los ODM está expresada en las consideraciones del Acuerdo Municipal mediante el cual se aprobó el Plan de Desarrollo, señalando que "las políticas, programas, subprogramas, estrategias y metas del Plan de Desarrollo "Chinchiná Compromiso de Todos" son coherentes con EI PND "y el proyecto de Plan Departamental de Desarrollo, los Objetivos del Milenio y la Agenda 2019, teniendo en cuenta el ejercicio de las respectivas competencias y observando los criterios de concurrencia, complementariedad y subsidiariedad ..."; No habiendo otra alusión a la forma en que efectivamente estos fueron considerados; aunque efectivamente, en las metas de resultado de los subprogramas se logra encontrar la afinidad entre algunos de los objetivos del milenio y las apuestas del municipio; principalmente en los sectores de educación y vivienda.

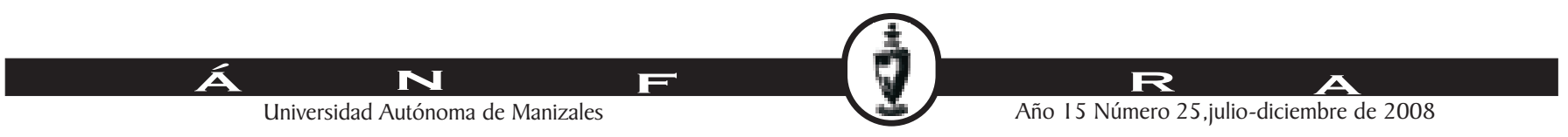


FILADELFIA 2008 - 2011 Juntos, Hagámoslo por Filadelfia

El Plan se estructura en una estrategia de sectores de inversión, siendo eje y base fundamental los ODM, partiendo desde su incorporación en la Misión del Plan y defininiedo subprogramas por sectores de acuerdo a cada objetivo.

\section{LA DORADA 2008 - 2011 Construyendo en paz la Dorada que todos queremos}

El plan de desarrollo se configura en cuatro líneas estratégicas en las que se distribuyen los sectores de desarrollo sobre los cuales la administración municipal diseña los programas de inversión. No se establece relación alguna en el proceso con los ODM; sin embargo algunas de las estrategias y metas podrían contribuir al avance en el logro de los objetivos.

LA MERCED 2008 - 2011 Por una Merced con Futuro

El Plan de Desarrollo estableció una relación importante con los ODM, inicialmente los referencia como elemento para la articulación del Plan y como insumo para la construcción del mismo y los indicadores hacen parte del diagnóstico como punto de partida.

La estrategia está fundamentada en la definición de programas sectoriales, donde se consideran algunas metas que apuntan a avanzar en el logro de los objetivos.

MANIZALES: Ciudad Internacional de conocimiento con oportunidades para todos

El objetivo central del plan es "la creación de las oportunidades sociales, culturales, económicas, territoriales, políticas e institucionales para ampliar las oportunidades de desarrollo humano integral para todos sus habitantes". Particularmente, los ODM son el primer referente estratégico y desde la misma definición del mismo se establece que "En todos los sectores del plan de desarrollo se encuentran incorporados los 8 objetivos de desarrollo del milenio, sus 18 metas y sus 48 indicadores y a partir de la alianza estratégica con el Programa de las Naciones Unidas para el Desarrollo, le haremos seguimiento a los alcances que para el 2011 tendrán cada uno de sus objetivos".

Al revisar las políticas del Plan, se encuentra que en ellas se asocian los objetivos pertinentes y son llevados a los programas y metas correspondientes.

MANZANARES Unido por el cambio

El plan de desarrollo está construido sobre 5 ejes estratégicos (Manzanares con actitud Social; Gestión para el mejoramiento integral; Fortaleciendo la productividad; Ciudadanía como modelo institucional; Inclusión con cavidad), en los cuales se establecen programas y para cada uno de ellos metas, donde los ODM tienen una incidencia importante, en razón a que fueron considerados como elemento fundamental del diagnóstico y los indicadores hacen parte de la línea base y de seguimiento.

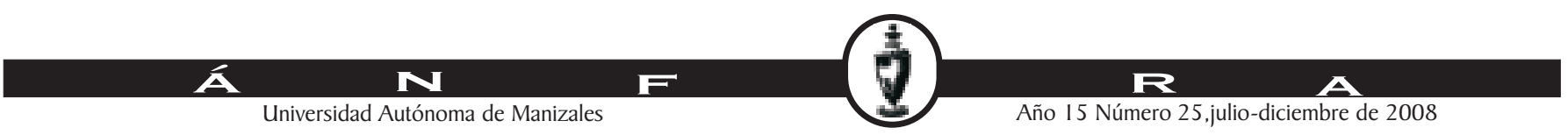


MARMATO 2008 - 2011 Hagamos de Marmato un municipio mejor

En el plan de desarrollo de Marmato se identifica un alto compromiso con los ODM; los cuales, además de ser considerados como referente de planeacion e incorporados en la línea base, tienen un tratamiento especial en el Sector de Modernización del Municipio, donde en el programa de Fortalecimiento Institucional se considera un subprograma de Municipalización de los Objetivos del Milenio, teniendo como meta la "Creación y operativizacion del Comité Municipal para el seguimiento de los ODM"; no obstante no se cuenta con una base de indicadores de meta clara para ese seguimiento.

MARQUETALIA Sociedad de Oportunidades

El plan de desarrollo referencia hacer parte del propósito Sociedad con alto nivel de convivencia.

Construido sobre cuatro áreas de desarrollo (Desarrollo Físico, Desarrollo Humano, Desarrollo Económico, Desarrollo Institucional), tiene en los ODM un referente para la planeación, considerando en cada uno de los programas que las conforman, el objetivo sobre el cual tiene incidencia y desarrollando la meta o indicador que permite el seguimiento.

Particularmente, en los propositos del plan, establece que "Los Objetivos de Desarrollo del Milenio ODM, serán los parámetros principales para la elaboración de estrategias, programas y proyectos que permitan un desarrollo integral de nuestra sociedad, en especial de nuestros niños, niñas y adolescentes"

MARULANDA 2008 - 2011 Ciudadanos por el porvenir de Marulanda

El plan de desarrollo está elaborado sobre 6 políticas (Social, Económica, Físico Territorial, Institucional, Ambiental y de Paz), en las cuales se definen sectores y por cada uno de ellos programas; donde los ODM tienen una consideración importante toda vez que son uno de los principales referentes de planeación y los indicadores que los evalúan hacen parte de la línea base del municipio y por cada uno de ellos se establecen las metas que busca la actual administración.

Adicionalmente, como anexo del Plan, se presenta una matriz de los programas que por cada sector se asocian a cada objetivo.

NEIRA 2008 - 2011 Todos por Neira

El plan se estructura sobre cuatro áreas de desarrollo (Social, Físico Territorial, Económico e Institucional), en las cuales se definen objetivos por sectores de inversión.

Los ODM hacen parte del conjunto de criterios, con el compromiso de dar cumplimiento a las metas ajustandose al CONPES 91 de 2005; no obstante no se desarrolla, por lo menos en el documento, una ampliación de éste proposito, aunque algunas metas si lo reflejan, principalmente en asuntos de educación.

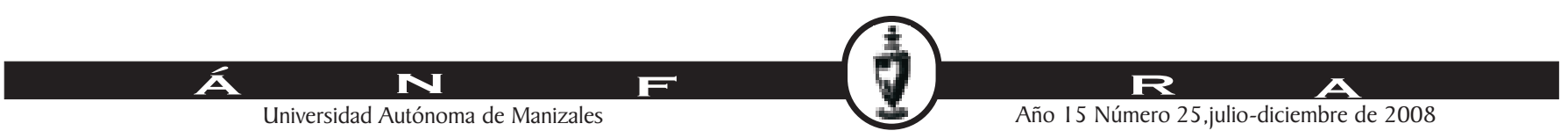


NORCASIA 2008 - 2011 Para que Norcasia siga progresando

La estructura del Plan está definida en 5 componentes (Educación, Bienestar Social, Calidad de Vida; Administrativo - Institucional; Económico; Desarrollo Físico, Urbanístico y Ambiental, y Participación y Organización), con 5 conectores (Planeación; Indicadores y Metas; Evaluación; Seguimiento y Control, y Comunicación).

No se registra ni se evidencia estructuralmente una referencia sobre los ODM, aunque en los programas, metas e indicadores se pueden encontrar algunos que apuntan al avance en el logro de los objetivos.

PACORA 2008 - 2011 Alcaldía con sentido social

Planteado en cinco áreas de Desarrollo (Social, Económico, Físico Territorial, Institucional, Subregional), no establece una referencia sobre los ODM para el proceso de formulación ó seguimiento; sin embargo en las metas de los programas se encuentran indicadores que de lograrse permitirán avanzar en el logro de los objetivos del milenio y en el del propio del plan, el cual busca "Lograr mayores niveles de equidad en la prestación de los servicios sociales a cargo de la Administración Municipal, atendiendo los sectores prioritarios de inversión a los cuales deben asignarse los ingresos del Sistema General de Participaciones y los recursos a gestionar"

\section{PALESTINA}

\section{PENSILVANIA}

\section{RIOSUCIO 2008 - 2011 Con Todos y Para Todos}

El plan de desarrollo establece seis áreas de desarrollo (Social; Económica; Físico Territorial; Derechos humanos, paz y convivencia; Institucional, Integración Regional y Proyectos Especiales), con sus respectivos sectores de trabajo; donde los ODM juegan un papel importante para el proceso de planificación y seguimiento toda vez que fueron asumidos como referente conceptual y línea base, pero además se estableció la creación de un programa para su municipalización proponiendo igualmente la conformación de un comité municipal para su seguimiento.

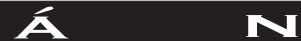

Universidad Autónoma de Manizales

\section{$\mathbf{R}$}

Año I5 Número 25,julio-diciembre de 2008 
RISARALDA 2008 - 2011 Unidos por el pueblo que soñamos

Con el objetivo de mejorar las condiciones de vida y capacidades de integración social de los risaralditas, se propone un Plan de Desarrollo estructurado como una estrategia que en cinco dimensiones de desarrollo y una estrategia institucional agrupan los sectores de inversión del municipio, donde los ODM cumplen un papel importante, toda vez que fueron aceptados como referente y los indicadores como línea base para la formulación del plan, además que fueron llevados a las metas propuestas por la administración en cada sector.

SALAMINA 2008 - 2011 Tiene un rumbo que queremos continuar

Los ODM hacen parte del conjunto de elementos del componente estratégico, referenciando principalmente su importancia hacia el compromiso para la superación de la pobreza, en un plan que está estructurado por áreas y sectores de inversion.

No se hace otra referencia a los Objetivos, llevando a que en un ejercicio de revisión de metas se pueda concluir que efectivamente algunas de ellas apuntan al avance en el logro de los mismos.

SAMANA 2008 - 2011 La Alcaldía de la Reconciliación

El plan de desarrollo tiene como referente conceptual y de línea base los ODM y los indicadores de su estado. De particular interés la incorporación del estado del municipio frenta a algunos objetivos en el capítulo de diagnóstico, donde se referencia la meta que se debe trazar el municipio para dar cumplimiento a ellos.

Definió en su estructura de Políticas, la implementación de un programa de municipalización de los Objetivos, sobre lo cual se espera un desarrollo de un proceso que permita hacer un seguimiento efectivo al proceso.

\section{SAN JOSÉ 2008 - 2011 Un San José para todos}

Buscando el reconocimiento del municipio como líder en el desarrollo del sector agropecuario, se estructuró un plan en tres dimensiones (Económica Productiva, Humana y Comunitaria, Ambiental y Físico Territorial), transversalizadas por una estrategia de desarrollo institucional, donde los ODM se consideraron como un referente para la formulación de programas.

Algunas metas son apuntan directamente al avance en el cumplimiento de los objetivos, principalmente las de salud y las de educación.

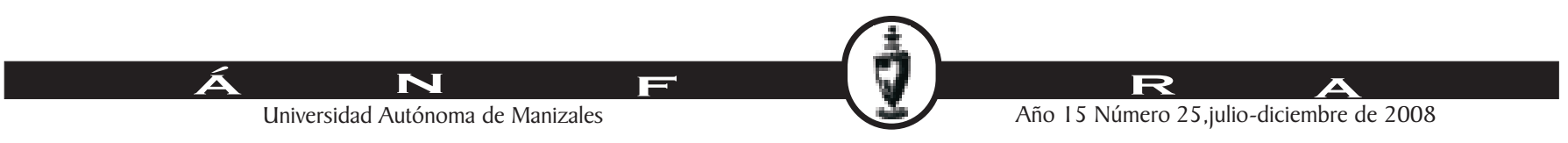


SUPÍA 2008 - 2011 Por el Respeto y el Bienestar de Todas y de Todos

El plan contiene una amplia visión, donde se establecen los propósitos de trabajo de la administración, siendo el desarrollo humano integral el principal objetivo, sobre tres ejes (Hacia un hábitat humano sostenible, hacia una sociedad educada, productiva y feliz, y camino a una sociedad participativa, solidaria y equitativa) y una línea de proyectos especiales, en los que se definen los programas de inversión del municipio.

No se hace una alusión a los ODM; algunos de los proyectos bandera podrían aceptarse como aporte para avanzar en el cumplimiento de los Objetivos.

VICTORIA 2008 - 2011 Por una Victoria unida trabajando por la paz

El Plan acepta los ODM como carta de navegación para la superación de la marginación social, en tal sentido asume los indicadores como línea base y formula metas para ajustarse al propósito nacional de cumplimento de ellos, en una estructura de sectores de inversión agrupados por dimensiones.

VILLAMARÍA 2008 - 2011 Todos por Villamaría

El plan de desarrollo presenta una estructura por sectores de inversión donde, particularmente en Educación, Salud y Desarrollo Rural, se hace referencia a la consideración de los ODM (Identificados como Plan del Milenio ó Metas del Milenio) para la definición de programas y ajuste de metas.

VITERBO 2008 - 2011 Progreso y calidad de Vida

El plan establece cinco sectores de inversión, donde se aceptan los ODM como un instrumento de referencia para la formulación. No se hace una alusión distinta a la anterior; sin embargo algunos programas y metas guardan relación con los Objetivos

\section{CALDAS 2008 - 2011 Para hacer de Caldas nuestra mejor empresa}

La Gobernación incorpora como criterio y referente estratégico de Planeación los ODM; incluso define su perspectiva de cooperación internacional bajo el enfoque de ellos. Sin embargo, en las formulaciones sectoriales, solo se considera explícitamente en el Objetivo de Agua Potable y Saneamiento Básico.

No se considera un programa especial para la departamentalización de los Objetivos, aunque algunas de las metas se podrían asociar al cometido de avanzar en el logro de estos.

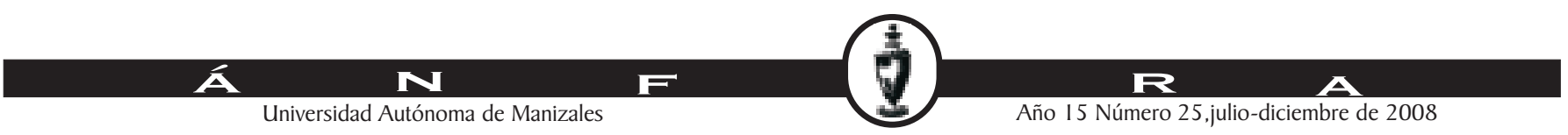




\section{Conclusiones}

La naturaleza y el espíritu con que se formularon los objetivos del milenio y sus correspondientes metas concuerdan de muchas maneras con la naturaleza y el espíritu del proceso de formulación de los planes de desarrollo territoriales; razón por la cual es, de algún modo, sencillo encontrar elementos de los planes que permitan establecer que hay una apuesta por el logro de los objetivos; no obstante se deben continuar los esfuerzos por bajar de la academia y las altas esferas institucionales a las comunidades el discurso y el propósito de los objetivos; tal vez sea necesario actualizar el CONPES 91 de 2005, de manera que explicite mayores responsabilidades a los entes territoriales, pero también mayores herramientas, recursos y elementos que descentralizacen el proceso y lo armonicen con cada realidad local.

En el ejercicio de revisión de los planes, se destaca el de Manizales, ya que evidencia un alto compromiso con las metas, apalancado en gran parte por el apoyo que recibe del PNUD. Se destacan igualmente los planes de La Merced, Aguadas, Risaralda, Manzanares, Marmato, Marquetalia, Victoria, Riosucio y Samaná y especialmente Marulanda, donde los indicadores sobre el estado municipal frente a los ODM fue considerado en su líneas base y para el caso de Marulanda se explicita en una matriz los programas que, dentro del plan, están orientados a avanzar en el logro de los Objetivos. No tiene ningún plan, tal vez con la excepción de Manizales, mas allá del seguimiento al propio indicador, una propuesta clara para la evaluación, articulación, focalización de los objetivos.

Reiterando la necesidad y oportunidad de liderar un proceso de Departamentalización y Municipalización de los Objetivos, con herramientas claras de formulación de proyectos, financiación de acciones y seguimiento y evaluación; desde quien corresponda, pero que podría ser desde la Gobernación en un acto de reivindicación, con apoyo de la academia, que ya lo está haciendo con la iniciativa de las cátedra del milenio; tal vez la primera actividad a acompañar sería una producción de una matriz por cada municipio donde se explicite cuales son los programas que podrían circunscribirse a cada Objetivo, un ejercicio que debería ser desarrollado, apropiado y sociabilizado por cada municipio, en cada municipio.

I gualmente, una responsabilidad que podríamos asumir, quienes estamos en la academia o vinculados de alguna manera a los entes territoriales, es avanzar en llevar la propuesta de los ODM a las comunidades, es frustrante, más allá de la hilaridad que produce, que en el desarrollo de este proceso, al consultar a un actor local en proceso de formación escolar media sobre su entendimiento de los Objetivos, su respuesta fuese que efectivamente es muy importante que la humanidad conquiste Marte y el espacio.

\section{Bibliografía}

ARANGO Maria Eugenia. Línea Base de los Objetivos del Milenio en el Departamento de Caldas. CEA UAM. Manizales, 2007.

PLANES DE DESARROLLO MUNICIPAL DE LOS MUNICIPIOS DE CALDAS VIGENCIA $2008-2011$

PLAN DE DESARROLLO DEPARTAMENTAL 2008 - 2011. Hagamos de Caldas Nuestra Mejor Empresa

CARTA ESTADÍSTICA DEL DEPARTAMENTO DE CALDAS 2007. Secretaria de Planeación Departamental.

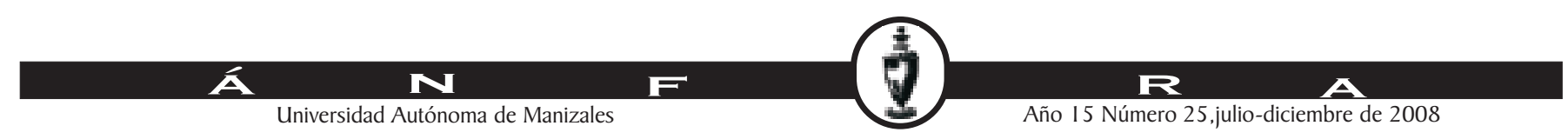

